\title{
Vergleichende Studie zum Verlauf röntgendichter Nervus-femoralis- \\ Katheter, die mit der In-plane- und der Out-of-plane-Technik angelegt wurden
}

\author{
INAUGURAL-DISSERTATION \\ zur Erlangung des Doktorgrades \\ der Medizinischen Fakultät der \\ Georg-August-Universität zu Göttingen
}

vorgelegt von

Jan Joschka Jodok Dracklé

aus

Hamburg

Göttingen 2019 
Dekan:

\section{Betreuungsausschuss}

Betreuer:

Ko-Betreuer:

\section{Prüfungskommission}

Referent:

Ko-Referent:

Drittreferent:
Prof. Dr. rer. nat. H.K. Kroemer

PD Dr. med. I. Bergmann

Prof. Dr. med. F. Petzke

Prof. Dr. Frank Petzke

Prof. Dr. Thomas Meyer

Datum der mündlichen Prüfung: 15.08.2019 
Hiermit erkläre ich, die Dissertation mit dem Titel „Vergleichende Studie zum Verlauf röntgendichter Nervus- femoralisKatheter, die mit der In-plane- und der Out-of-plane-Technik angelegt wurden" eigenständig angefertigt und keine anderen als die von mir angegebenen Quellen und Hilfsmittel verwendet zu haben.

Göttingen, den 


\section{Inhaltsverzeichnis}

Abbildungsverzeichnis .........................................................................................II

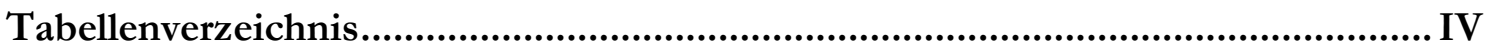

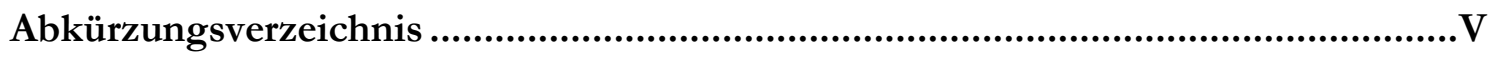

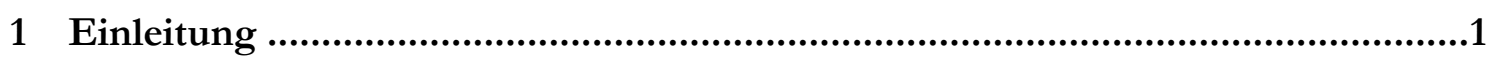

1.1 Allgemeine periphere Leitungsbahnanästhesie ..........................................................................2

1.2 Der Verlauf und die Innervation des Nervus femoralis..................................................................

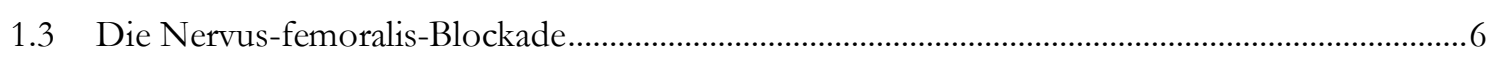

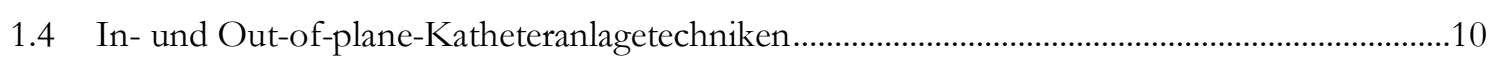

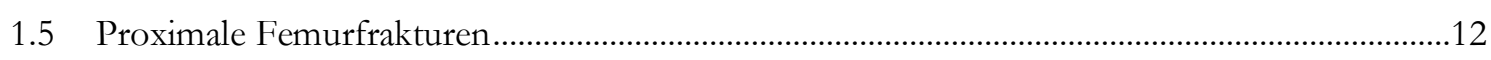

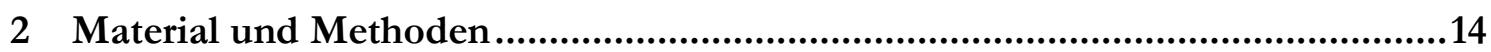

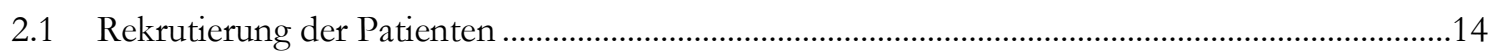

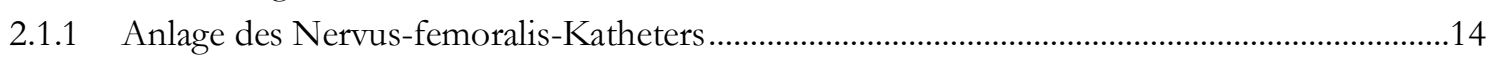

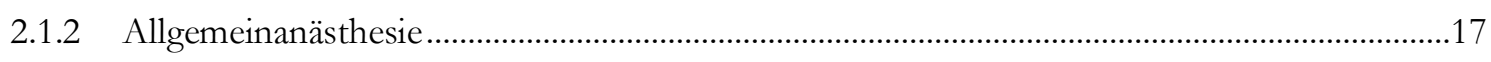

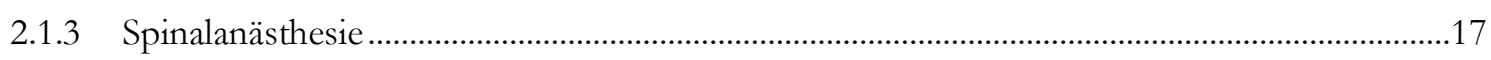

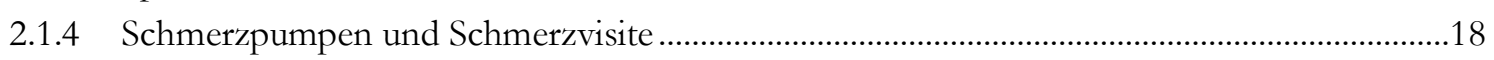

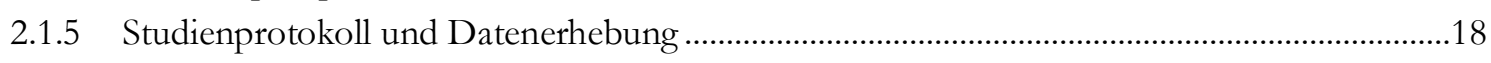

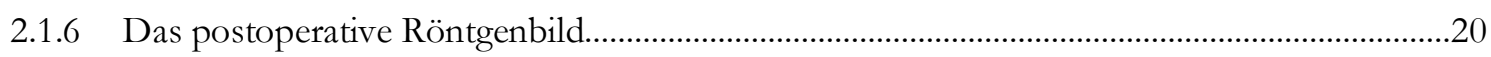

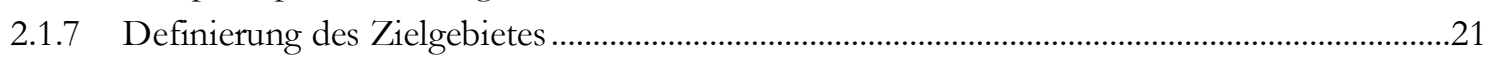

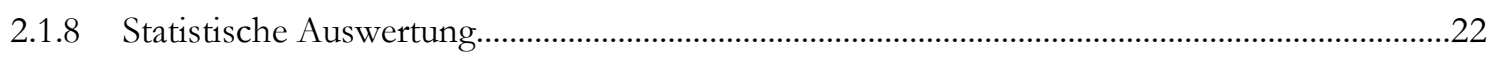

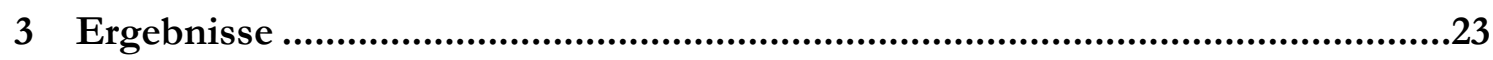

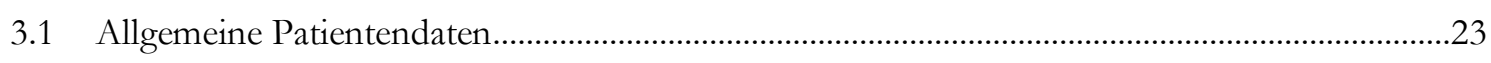

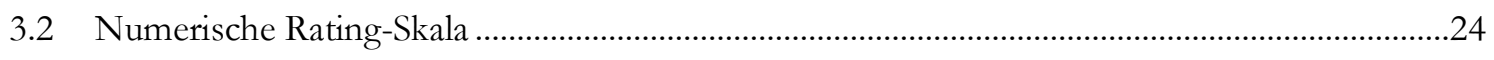

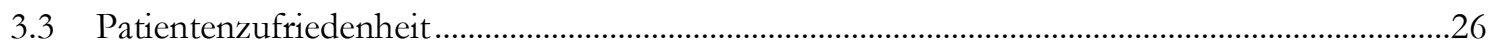

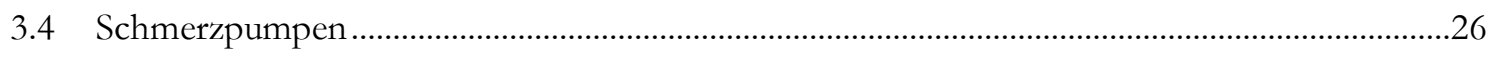

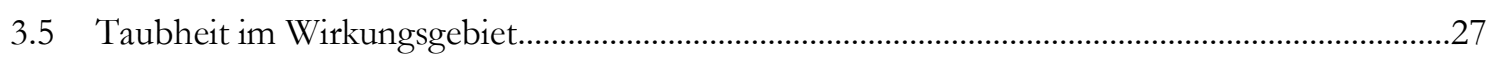

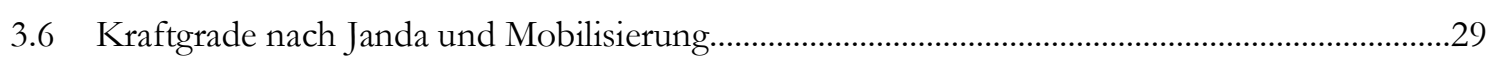

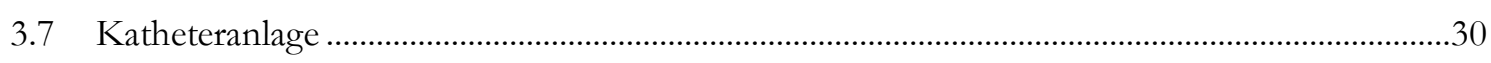

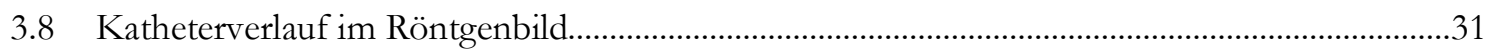

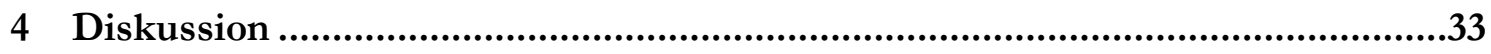

4.1 Bewertung der eigenen Ergebnisse .........................................................................................

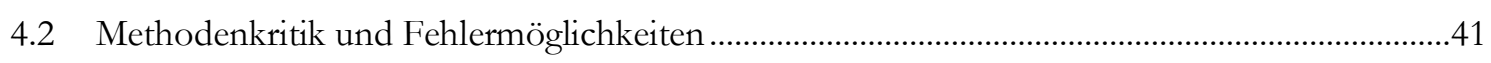

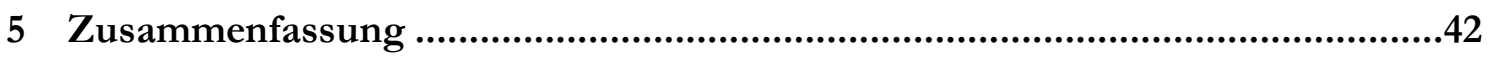

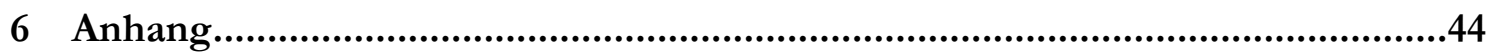




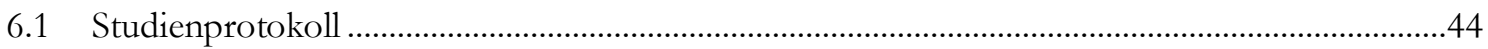

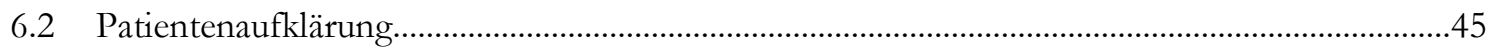

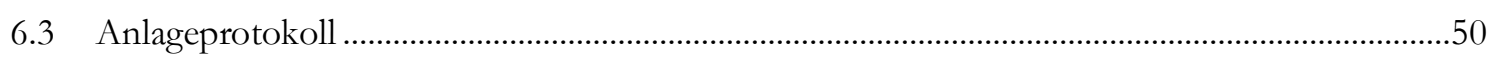

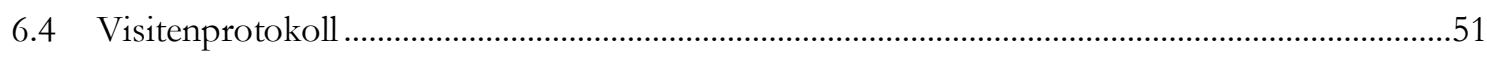

7 Literaturverzeichnis ......................................................................52 


\section{Abbildungsverzeichnis}

Abbildung 1: Lacuna musculorum et vasorum...................................................................................

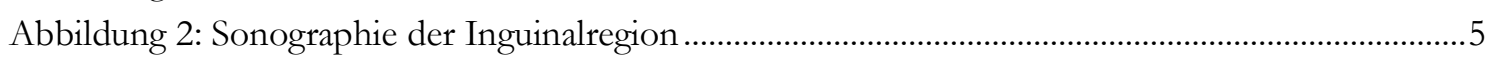

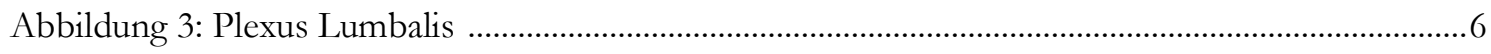

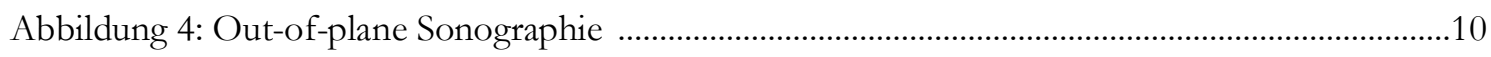

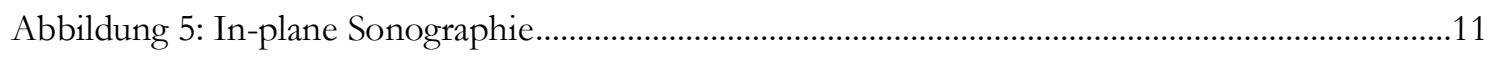

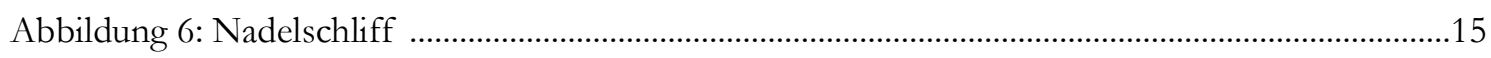

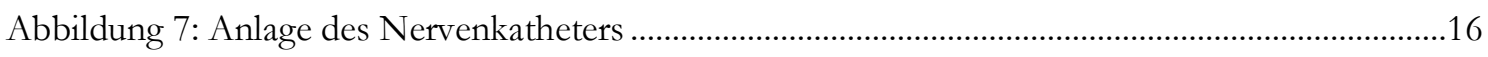

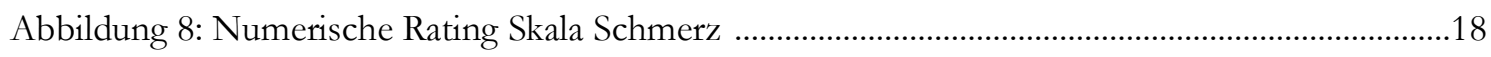

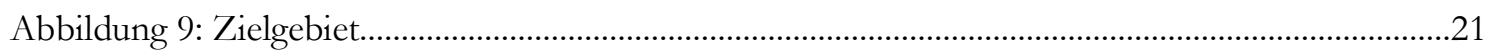

Abbildung 10: Verteilung der Katheterspitzen in der IP- und in der OOP-Gruppe .............................31

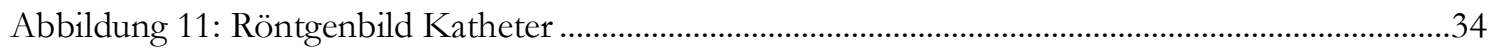




\section{Tabellenverzeichnis}

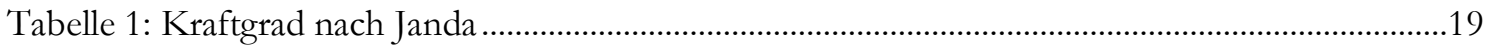

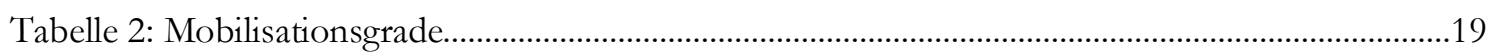

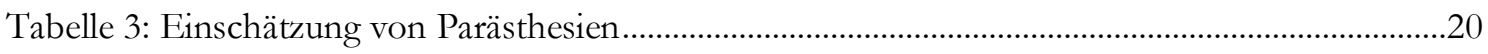

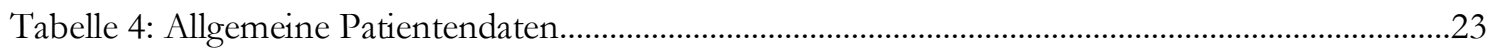

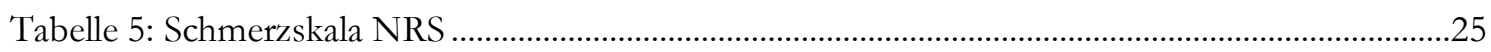

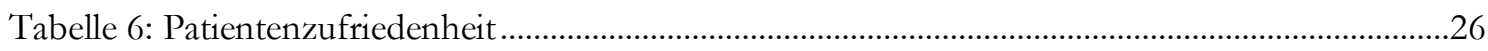

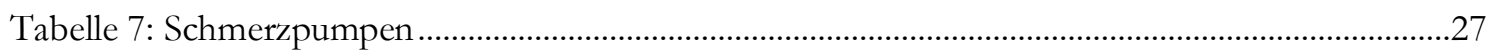

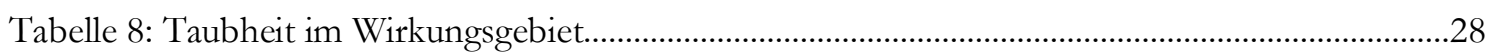

Tabelle 9: Kraftgrade nach Janda und Mobilisierungsgrade ..............................................................30

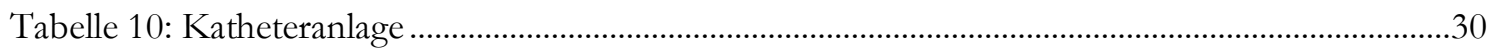

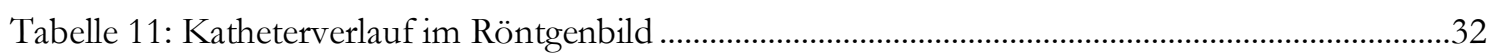




\section{Abkürzungsverzeichnis}

$\mathrm{AO}$

Ant

ASA

DHS

DIVS

DVO

G

h

IP

KG

Lat

MAD

Med

$\mathrm{mmHG}$

NHS

NRS

NSAR

OP

OOP

OS

PCA

PFN

POD
Arbeitsgemeinschaft für Osteosynthese-

fragen

Anterior

American Society of Anesthesiologists

Dynamische Hüftschraube

Deutsche Interdisziplinäre Vereinigung für Schmerztherapie

Dachverband Osteologie

Gauge

Stunde (bour)

In plane

Körpergewicht

Lateral

Mittlerer arterieller Druck

Medial

Millimeter-Quecksilbersäule

National Health System

Numerische Rating-Skala

Nicht-steroidale Antirheumatika

Operation

Out-of-Plane

Oberschenkel

Patient-controlled-analgesia

Proximaler Femurnagel

Postoperative day 
PONV

p.o.

SAX

TEP

TIVA
Postoperative nausea and vomiting

peros

short axis

Totalendoprothese

Totale intravenöse Anästhesie 


\section{Einleitung}

Die kontinuierliche Blockade des Nervus femoralis ist ein etabliertes Verfahren zur perioperativen Analgesie unter anderem bei Patienten mit hüftgelenksnahen Operationen, das sowohl mit Hilfe der Nervenstimulation als auch mit der Sonographie durchgeführt werden kann (Guay et al. 2016). Bei der ultraschallgesteuerten Anlagetechnik von Nervenkathetern kann die Nadelspitze bis direkt an den Zielnerven innerhalb der anatomischen Verhältnisse in Echtzeit kontrolliert werden (Griffin und Nicholls 2010). Prinzipiell stehen hierfür mit der In-plane (IP) und der Out-of-plane (OOP) geführten Nadelpunktionstechnik zwei gleichwertige ultraschallgesteuerte Verfahren zur Verfügung, die jedoch naturgemäß spezifische Vor- und Nachteile mit sich bringen (Griffin und Nicholls 2010; Ilfeld et al. 2011). Bei der üblichen Darstellung in der kurzen Achse (short axis/SAX) wird bei der OOP-Technik die Nadel senkrecht zur Ultraschallebene und parallel zum Nervenverlauf geführt, sodass der Nervenkatheter über die Punktionsnadel entlang des Zielnerven vorgeschoben werden kann und theoretisch direkt am Zielnerv zu liegen kommt (Ilfeld et al. 2011). Hierbei ist die Visualisierung der Nadelspitze häufig schwieriger als mit Hilfe der IP-Technik, bei der sich die Nadel innerhalb der Ultraschallebene in ihrer gesamten Länge kontinuierlich visualisieren lässt. Somit können bei der IP-Technik akzidentelle intraneurale oder intravasale Fehlinjektionen vermeintlich besser vermieden werden. Allerdings wird hierbei der Nervenkatheter über die Punktionsnadel senkrecht auf den Verlauf des Nervs vorgeschoben, sodass eine Fehlposition des Nervenkatheters oder sogar eine Perforation des Nervs denkbar sind. Unzureichende Analgesie oder Nervenschäden wären die Folge. Allerdings erwiesen sich für femorale Nervenkatheter die IP- und die OOP-Anlagetechnik hinsichtlich postoperativer Analgesie in den bisher durchgeführten Vergleichsstudien als gleichwertig (Wang et al. 2010; Fredrickson und Danesh-Clough 2013).

Grundsätzlich ist jedoch die direkte Platzierung der Katheterspitze an den Zielnerven mit der traditional catheter-through-needle insertion technique bei beiden Anlagetechniken noch nicht sicher möglich (Elsharkawy et al. 2016). Zudem werden Dislokationsraten femoraler Nervenvenkatheter nach Mobilisation von bis zu 25\% beschrieben (Marhofer et al. 2013), sodass ein sekundäres Katheterversagen in den ersten postoperativen Tagen durchaus häufig beschrieben wird (20-40\%, Grant 2001). Zwar sind Methoden, den Verlauf von Nervenkathetern und die Lage der Katheterspitze bei Anlage zu kontrollieren, zunehmend Gegenstand aktueller Studien (Elsharkawy et al. 2016), jedoch liegen bisher keine Daten 
vor, ob femorale Nervenkatheter in Abhängigkeit von den beiden Anlagetechniken unterschiedliche Verläufe nehmen oder wo deren Spitze zu liegen kommt.

Wir haben in dieser Studie Verlauf und Lage der Spitze femoraler Nervenkatheter miteinander verglichen, die ultraschallgesteuert in der Out-of-plane oder In-plane geführten Nadelpunktion in SAX angelegt wurden. Auf der Basis von anterior-posterioren Röntgenaufnahmen des Hüftgelenks am zweiten postoperativen Tag wurde der Verlauf von röntgendichten Nervenkathetern im Verhältnis zur proximodistalen Achse des Femurs bestimmt. Des Weiteren wurde der Abstand der Katheterspitze zu einer zuvor definierten Zielregion mit Projektion über dem inguinalen Anteil des Nervus femoralis ins Verhältnis gesetzt. Neben der visuellen Komponente wurden gleichfalls qualitative Daten der Nervenblockade erfasst. So wurden nebst der Inzidenz und Intensität des postoperativen Schmerzes mittels der numerischen Rating-Skala (NRS) auch die Qualität der Nervenblockade bis zum zweiten postoperativen Tag dokumentiert. Ziel dieser Studie war es, die Hypothese zu überprüfen, ob mit einer der beiden Anlagetechniken (IP vs OOP) die Nervenkatheterspitze häufiger innerhalb der vordefinierten Zielregion nach proximal gerichtet platziert werden kann, und ob sich hieraus unterschiedliche Effekte bei der postoperativen Analgesie ergeben.

\subsection{Allgemeine periphere Leitungsbahnanästhesie}

Die periphere Leitungsanästhesie blockiert die Nervenleitung eines bestimmten Nervs. Dieser wird durch die Applikation von Lokalanästhetika in seiner jeweiligen Funktion reversibel gehemmt. Hiervon sind die Spinal- und Epiduralanästhesie sowie die intravenöse Regionalanästhesie abzugrenzen. Die Blockade der Nervenleitung und der damit verbundenen Funktionen erfolgt durch die Applikation von Lokalanästhetika in den Bereich des jeweiligen Nervs. Der Wirkmechanismus erfolgt durch die Blockade des schnellen Natrium Einstroms während der Erregungsleitung.

Lokalanästhetika werden gemäß ihrer chemischen Struktur in Aminoamide und Aminoester (Amid- und Ester-Typ) unterteilt. Zur Gruppe der Aminoester-Lokalanästhetika gehören die Substanzen Kokain, Procain, Benzocain, Tetracain und Chlorprocain. Zur Gruppe der Aminoamid-Lokalanästhetika zählen Lidocain, Ropivacain, Bupivacain, Prilocain, und Mepivacain. Für die Blockade des Nervus femoralis sind, dem Klinikstandard entsprechend, vor allem Ropivacain und Prilocain von Bedeutung.

Bei diesen Lokalanästhetika handelt es sich mit Ausnahme des Benzocains um schwache Basen. Die Lokalanästhetika diffundieren zunächst in ungeladener Form durch die Zell- 
membran. Die geladene kationische Wirkform entsteht nach Dissoziation innerhalb der Nervenzelle. Diese geladene kationische Wirkform bindet an die alpha1-Untereinheit des Natriumkanals, wodurch dieser blockiert wird. In der Folge kommt es zur Abnahme des Aktionspotentials der Leitungsgeschwindigkeit und der Anstiegsgeschwindigkeit des Aktionspotentials, sowie zu einer Erhöhung der Depolarisationsschwelle und einer Verlängerung der Refraktärzeit. Die Bindung an den Natriumkanal ist reversibel. Die Eigenschaften der Lokalanästhetika wie Wirkdauer, Wirkungspotenz und Anschlagszeit werden durch verschiedene Faktoren bestimmt. Eine hohe Lipidlöslichkeit sowie eine hohe Prteinbindung der Lokalanästhetika haben eine verlangsamte Anschlagszeit, eine verlängerte Wirkdauer und eine höhere Potenz zur Folge. Dies ist etwa bei dem in dieser Studie verwendeten Ropivacain 0,75\% der Fall. Hingegen bedingen eine niedrige Lipidlöslichkeit und eine schwache Proteinbindung einen schnelleren Wirkungseintritt, eine kurze Wirkdauer und eine schwächere Potenz des Lokalanästhetikums. Der Abbau der Lokalanästhetika erfolgt für den Amid-Typ in erster Linie in der Leber über Cytochrom p450 und für den Ester-Typ über die Plasmacholinesterase (Dullenkopf und Borgeat 2003).

\subsection{Der Verlauf und die Innervation des Nervus femoralis}

Der Nervus femoralis entspringt dem Plexus lumbosacralis (L1-L4). Er trägt als peripherer Nerv sowohl sensible als auch motorische Anteile. Er tritt zunächst lateral des Musculus psoas major aus. Dort verläuft er unter der Fascia iliaca zwischen dem Musculus psoas major und dem Musculus iliacus, bevor er in die Lacuna musculorum eintritt. Diese Struktur unterhalb des Leistenbandes wird durch eine Septierung der Fascia lata gebildet. Diese Septierung bildet die Lacuna musculorum und medial dazu die Lacuna vasorum. 


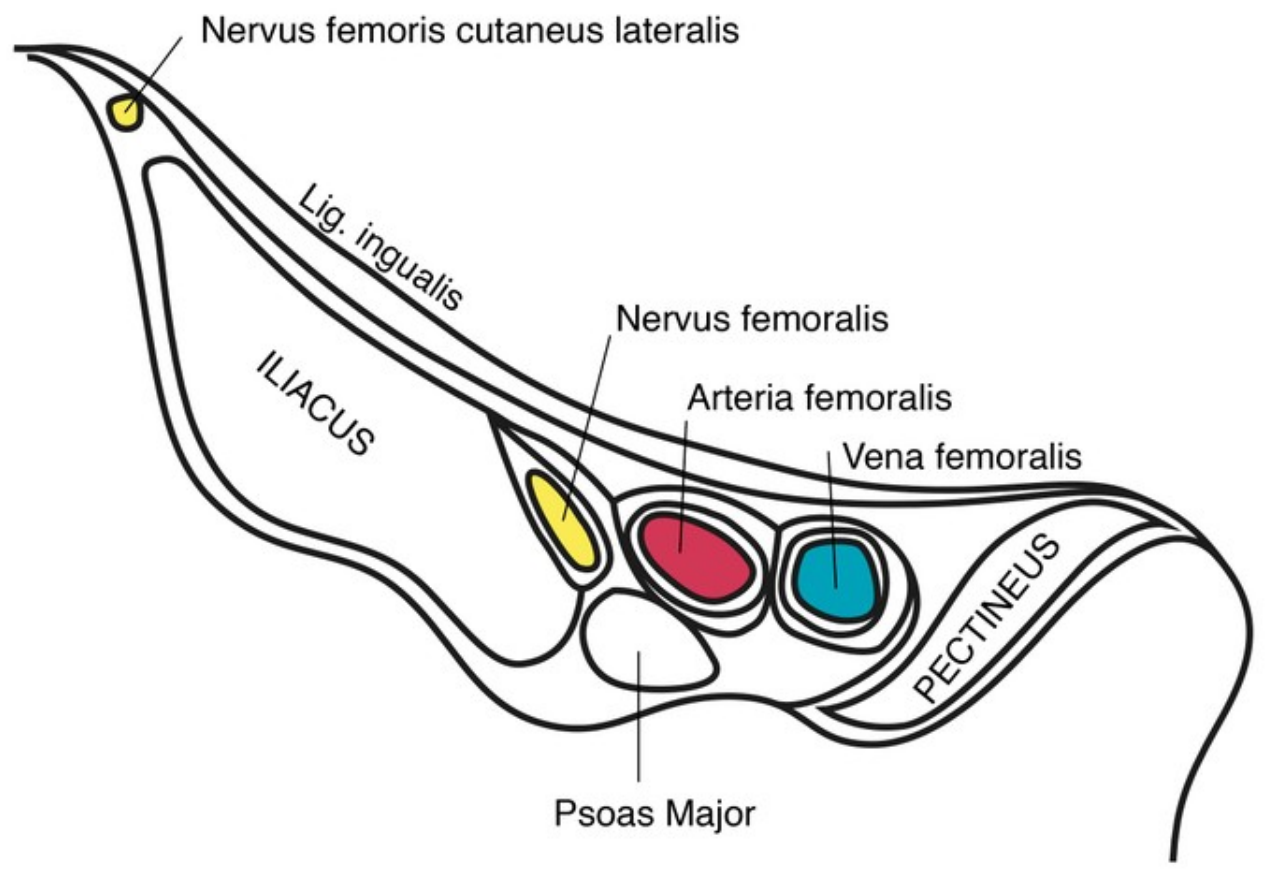

Abbildung 1: Lacuna musculorum et vasorum

Die Lacuna musculorum bildet sich aus drei Anteilen. Ventral wird sie vom Ligamentum inguinale, dorsal durch den oberen Rand des Os ilium und medial durch den Arcus iliopectineus begrenzt. Durch sie hindurch verlaufen der Nervus femoralis, der Musculus iliopsoas sowie der Nervus cutaneus femoris lateralis (s. Abbildung 1). Wichtig für die Orientierung bei einer sonographischen Navigation ist die Anordnung der Strukturen in der Region des Leistenbandes, hier liegen von medial nach lateral zunächst die Vena femoralis, die Arteria femoralis und am lateralen Ende der Nervus femoralis (s. Abbildung 2). 


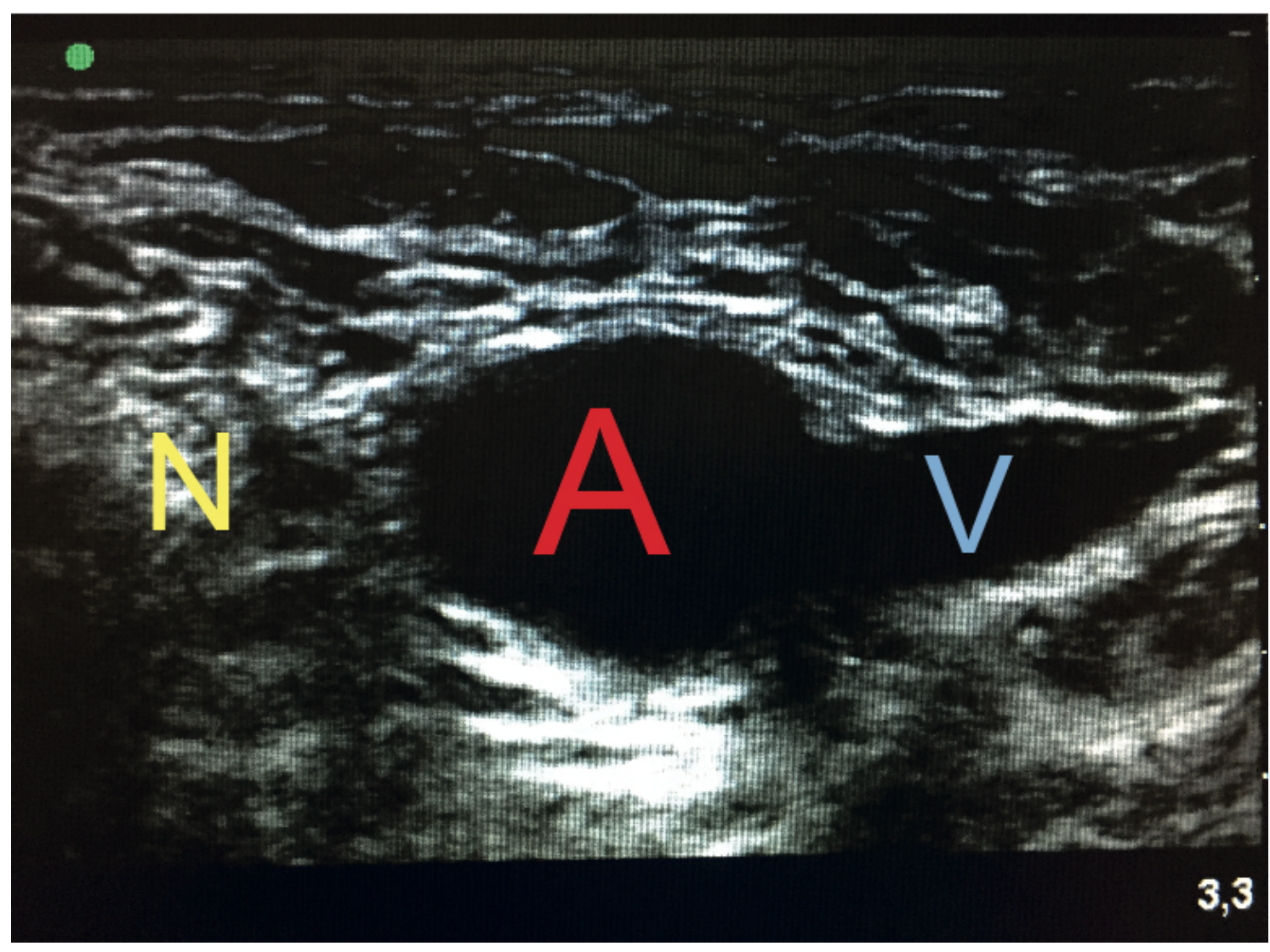

Abbildung 2: Sonographie der Inguinalregion

Nach dem Durchtritt durch die Lacuna musculorum innerviert der Nervus femoralis die Extensoren des Oberschenkels. Dazu gehören der Musculus quadriceps femoris und der Musculus sartorius. Die sensible Innervation des ventralen Oberschenkels erfolgt durch die Rami cutanei anteriores. Anschließend bildet der Nervus femoralis seinen sensiblen Endast, den Nervus saphenus, aus. Dieser verläuft am Oberschenkel gemeinsam mit der Arteria und Vena femoralis unter dem Musculus sartorius. Im Adduktorenkanal liegt er dem Musculus adductor longus auf. In Höhe der Patella gibt er den oberflächlichen Hautnerv (Ramus infrapatellaris) ab. Am Unterschenkel läuft der Nervus saphenus begleitend zur Vena saphena magna und innerviert so die Innenseite des Unterschenkels und des Fußes sensibel. Der Nervus saphenus besitzt keine motorischen Anteile.

Für die sensible Innervation des lateralen Oberschenkels ist der Nervus cutaneus femoris lateralis verantwortlich. Dieser speist sich aus den Nervenwurzeln der Segmente L2 und L3 (s. Abbildung 3). Er enthält allgemeine somatosensible Fasern und zieht ebenfalls durch die Lacuna musculorum, um anschließend zum lateralen Oberschenkel zu gelangen (Drake et al. 2015). 


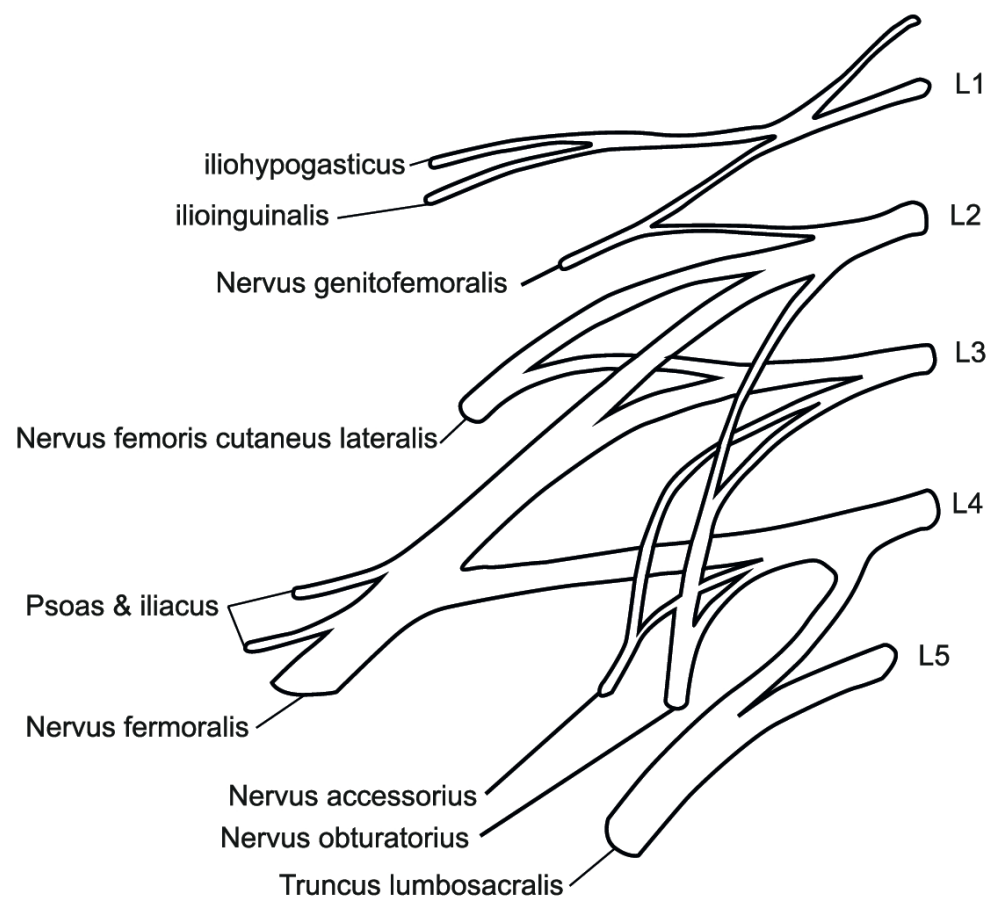

Abbildung 3: Plexus lumbalis

\subsection{Die Nervus-femoralis-Blockade}

Die Blockade des Nervus femoralis ist eine sichere und weit verbreitete Technik zur Unterstützung der Anästhesie und der postoperativen Analgesie nach Hüftoperationen (Finlayson und Underhill 1988; Fournier et al. 1998; Fletcher et al. 2003; Beaudoin et al. 2010). Die perioperative Blockade des Nervus femoralis wird deshalb von der DIVS in der S3- „Leitlinie Behandlung akuter perioperativer und posttraumatischer Schmerzen“ (2009) für alle Patienten mit einer Femur- bzw. einer Oberschenkelhalsfraktur empfohlen.

Im Jahre 1973 beschrieben Winnie et al. eine inguinale paravaskuläre Technik für die sogenannte 3-in-1 Blockade. Hierbei konnte gezeigt werden, dass die Blockade des Nervus femoralis, des Nervus obturatorius und des Nervus cutaneus femoralis durch die Injektion einer einzelnen Dosis Lokalanästhetikums möglich ist (Winnie et al. 1973).

Aus dieser Technik heraus ergibt sich ein weites Feld von Anwendungsmöglichkeiten für Operationen und Schmerzmanagement der unteren Extremität. Zu den Indikationen des 3in-1 Blockes bzw. einer reinen Nervus-femoralis-Blockade gehören Operationen der Hüfte und des ventralen Oberschenkels sowie in Kombination mit einer Blockade des Nervus ischiadicus alle weiteren Operationen der unteren Extremität.

Zur Analgesie findet die Technik weiterhin Anwendung bei Hüftfrakturen, bei Stumpfschmerzen nach Amputationen und bei der Mobilisierungstherapie bei Knie- und Hüftge- 
lenken (Marhofer et al. 1997). Besonders bei Patienten mit einer signifikanten Opiattoleranz, Opiatallergie und Co-Morbiditäten kommt der Blockade des Nervus femoralis ein hoher Stellenwert zu (Christos et al. 2010).

In einer Studie von Antonis et al. konnte gezeigt werden, dass bei einer Behandlung mit einem Nervus-femoralis-Block eine starke Abnahme der Schmerzen auf der visuellen Analogskala sowie eine Reduktion der Opioidbedürftigkeit zur Schmerzkontrolle besteht. Während der vier-Stunden-Morphiumgebrauch in der Gruppe, welche einen Nervenblockade erhielt, bei nur 5,5 mg lag, wurden in der Kontrollgruppe 15,5 mg benötigt (Antonis et al. 2006). Auch die benötigte Zeit, die notwendig ist zum Erreichen des geringsten Schmerzgrades, ist signifikant kürzer (2,88 gegenüber 5,81 Stunden) bei der Behandlung mit einem Nervenblock gegenüber der Behandlung mit intravenösem Morphin (Fletcher et al. 2003). Im Zusammenhang mit der Wahrscheinlichkeit, ein postoperatives Delirium zu entwickeln, scheint postoperativer Schmerz ein wichtiger Faktor zu sein (Lynch et al. 1998; Vaurio et al. 2006). Das postoperative Delirium ist eine extrem häufige postoperative Komplikation und tritt bei bis zu 60\% der Patienten auf (Parikh und Chung 1995).

Einer Studie von Kinjo et al. zufolge stellt das Schmerzmanagement für die Voraussehbarkeit des postoperativen Deliriums einen wichtigen Faktor dar. Insbesondere konnte gezeigt werden, dass die Rate des postoperativen Deliriums durch den Einsatz von Nervusfemoralis-Blockaden gesenkt werden konnte (Kinjo et al. 2012).

Für die postoperative Analgesie stehen sowohl Single-Shot als auch kontinuierliche Katheterverfahren zur Verfügung. Aufgrund der großen Wundfläche und des Ausmaßes der Operation werden für die Analgesie bei proximalen Fermurfrakturen zumeist die kontinuierlichen Katheterverfahren bevorzugt.

Der Einsatz von Bedside-Ultraschall ermöglicht die präzise Darstellung der neurovaskulären Anatomie. Aufgrund der ubiquitären Verfügbarkeit von Ultraschallgeräten und durch den routinierten Gebrauch der modernen Sonographie, wie z. B. für die Zuhilfenahme bei der Anlage eines zentralen Venenkatheters, ist der Einsatz von Ultraschall bei einer Nervus-femoralis-Blockade für das Personal eine leicht zu erlernende und auch in der Akutsituation gut praktikable Technik.

Es konnte gezeigt werden, dass durch die Zuhilfenahme eines Ultraschallgerätes der Wirkungseintritt des Lokalanästhetikums verkürzt und die Qualität der Betäubung verbessert werden kann. Vor allem unter schwierigen anatomischen Verhältnissen, wie z. B. Adiposi- 
tas, voroperierten Patienten oder bei Patienten mit anatomischen Anomalien, bietet das ultraschallgestützte Verfahren einen klaren Vorteil gegenüber der herkömmlichen „Landmark“"Technik (Marhofer et al. 1997).

Auch bietet der Einsatz von Ultraschall einen guten Schutz vor der versehentlichen Injektion von Lokalanästhetikum in die femoralen Gefäße, was lebensbedrohliche Konsequenzen nach sich ziehen kann. Bupivacain, das am häufigsten benutzte Lokalanästhetikum, birgt beispielsweise die Gefahr ventrikulärer Arhythmien, Hypotonien und Atemstillstand, wenn es fälschlicherweise in die systemische Zirkulation injiziert wird (Beaudoin et al. 2010).

Im operativen Bereich kann eine Nervus-femoralis-Blockade auch mittels eines Nervenstimulators durchgeführt werden. Allerdings sind Nervenstimulatoren in der Akutsituation meist nicht verfügbar und bedeuten einen zusätzlichen Kostenaufwand sowie eine spezielle Schulung für das Personal. Die Verwendung eines Nervenstimulators, ist bei der Versorgung frischer Frakturen auch deshalb kritisch zu sehen, da durch die elektrische Stimulation des Nervens schmerzhafte Muskelkontraktionen hervorgerufen werden. Im Hinblick auf den Wirkungsbeginn und die Menge an verwendetem Lokalanästhetikum scheint ohnehin der ultraschallgestützte Nervenblock vorteilhafter zu sein (Marhofer et al. 1997).

Durch die DIVS wurden relative und absolute Kontraindikationen definiert. Zu den absoluten Kontraindikationen zählen eine Ablehnung durch den Patienten, eine Infektion an der Punktionsstelle sowie eine bekannte Allergie auf Lokalanästhetika. Das Vorhandensein einer Gerinnungsstörung sowie eine vorbestehende Nervenschädigung gelten als relative Kontraindikationen für die Anlage einer Nervenblockade. Das Vorbestehen einer Nervenschädigung erfordert eine sorgfältige Dokumentation der Schädigung (DIVS Leitlinie 2009).

Generell können durch den Gebrauch von Lokalanästhetika verschiedene Nebenwirkungen induziert werden. Hierzu zählen allergische Reaktionen, die häufiger bei Lokalanästhetika vom Aminoester-Typ auftreten. Weiterhin haben Benzocain und Prilocain dosisabhängig das Potential zur Bildung von Methämoglobin durch Oxydation von HämoglobinEisen (Dullenkopf und Borgeat 2003). Auf systemischer Ebene besteht die Gefahr von Nebenwirkungen, vor allem durch die versehentliche intravasale Applikation von Lokalanästhetika. Bei einer intravasalen Applikation besteht die Gefahr der Wirkung auf Natriumkanäle an Herz-, Muskel- und Nervenzellen im Gehirn. Das Ausmaß der Symptome wird hierbei durch die Plasmakonzentration des Lokalanästhetikums bestimmt. 
Eine Vielzahl von Symptomen kann hierdurch hervorgerufen werden. Dazu zählen neurologische Symptome wie periorales Kribbeln oder Taubheitsgefühle, Kopfschmerzen und Benommenheit, Schwindel und Ohrensausen, Seh- und Sprechstörungen, Muskelfaszikulationen und Verwirrtheit. Schwerere Symptome bis hin zur Bewusstlosigkeit, Krampfanfälle und Koma mit Atemstillstand sind möglich.

Kardiale Symptome treten nach den ersten neurologischen Symptomen auf und bieten einen extrem heterogenen Symptomkomplex. Es kann zu Hyper- und Hypotension kommen, EKG-Veränderungen wie Tachy- und Bradykardien, AV-Dissoziationen können auftreten und Myokardischämien bis hin zur Asystolie mit Kreislaufstillstand (Dullenkopf und Borgeat 2003; Zink und Graf 2003).

Die Wahrscheinlichkeit einer durch eine Nervus-femoralis-Blockade induzierte periphere Neuropathie wird mit ca. 3/10000 angegeben (Auroy et al. 2002).

Auf lokaler Ebene sind in höheren Dosierungen Neurotoxizität und Myotoxizität von Lokalanästhetika beschrieben worden (Heavner 2007).

Es wurden des Weiteren direkte Nervenverletzungen und intraneurale Injektionen von Lokalanästhetikum beschrieben, allerdings ohne ernsthafte Folgeerscheinungen zu hinterlassen (Schafhalter-Zoppoth et al. 2004). Generell können Nervenläsionen bei einer peripheren Regionalanästhesie durch direkte toxische Effekte der Lokalanästhetika, durch Nervenkompression bei schneller Applikation, durch Ödembildung, durch eine Verletzung des Perineuriums, durch Ischämien oder durch eine intrafaszikuläre Injektion hervorgerufen werden (Hogan 2008). Permanente Nervenschäden wurden nur in Einzelfällen beschrieben, und die Inzidenz von Nervenläsionen nach einem Regionalanästhesieverfahren wird je nach Studie mit 0,02\% bis 0,04\% angegeben (Auroy et al. 2002; Capdevila et al. 2005; Brull et al. 2007; Barrington et al. 2009).

Variationen in der interindividuellen Effektivität der Nervenblockade lassen sich unter anderem auf unterschiedliche sensorische Innervationen der Hüfte zurückführen. Der Femurschaft sowie die distale Epiphyse werden fast ausschließlich durch den Nervus femoralis innerviert. Anatomische Studien zeigen allerdings, dass die Hüfte einer größeren Variation hinsichtlich der Innervation unterliegt. Hauptsächlich wird die Hüfte vom Nervus femoralis, Nervus obturatorius und dem Nervus lateralis cutaneus innerviert, gelegentlich kommt ein Teil der sensiblen Innervation jedoch vom Nervus glutealis superior und vom Nervus ischiadicus (Birnbaum et al. 1997). 
Die Inzidenz einer behandlungsbedürftigen Artherofibrose bei totaler Knieartheroplastie kann durch den Einsatz einer Nervus-femoralis-Blockade gesenkt werden. Dieser Effekt ist wahrscheinlich auf die verbesserte Mobilisierbarkeit der Patienten aufgrund der verbesserten postoperativen Analgesie zurückzuführen und könnte auch auf eine Hüftoperation zutreffen (Sharma et al. 2010).

\subsection{In- und Out-of-plane-Katheteranlagetechniken}

Bei der Out-of-plane-Technik zur Anlage eines Nervus-femoralis-Katheters wird die Nadel entgegen der horizontalen Achse des Ultraschallkopfes eingebracht (s. Abbildung 4). Die Stichrichtung entspricht hierbei dem natürlichen Verlauf des Nervus femoralis. Die Nadel ist als hyperdenser Punkt auf dem Ultraschallbild zu Erkennen.

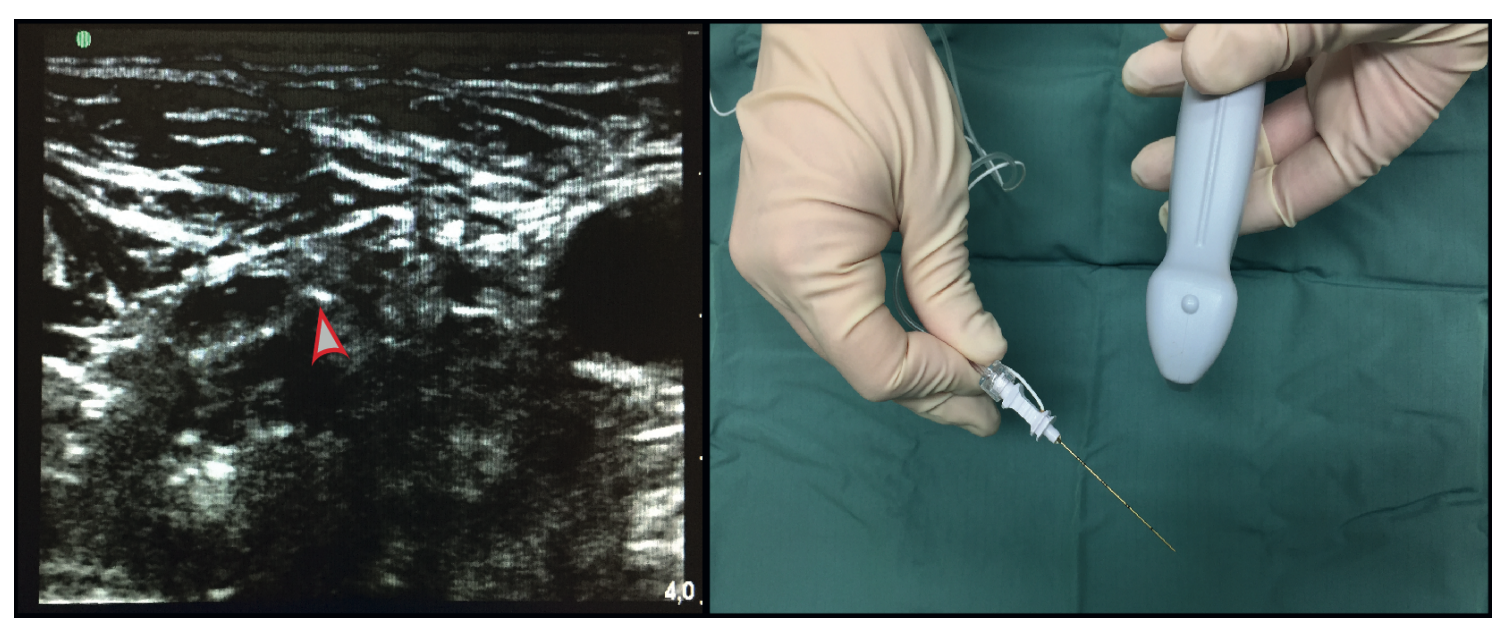

Abbildung 4: Out-of-plane-Sonographie

Für die In-plane Anlage erfolgt die Stichrichtung hingegen entlang der Achse des Ultraschallkopfes. Die Nadel trifft somit in einem $90^{\circ}$-Winkel auf den Nerven. Die Nadel ist in ihrer kompletten Länge einsehbar (s. Abbildung 5). 


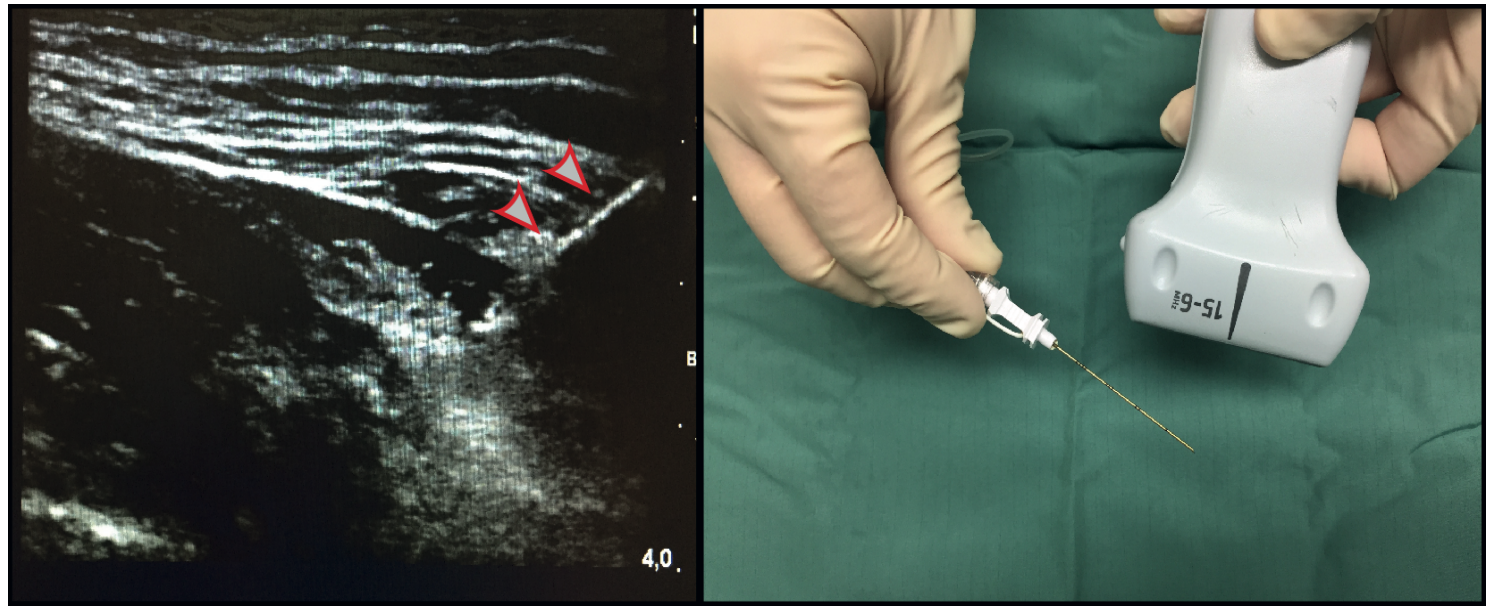

Abbildung 5: In-plane-Sonographie

In- bzw. Out-of-plane Anlagetechniken sind zwei bislang gleichwertig anerkannte Verfahren zur Anlage eines Nervus-femoralis-Katheters und werden in der klinischen Routine gleichermaßen angewandt (Fredrickson und Danesh-Clough 2013). Der Einsatz der beiden etablierten ultraschallgeführten Anlagetechniken für den Nervus-femoralis- Katheter bietet in der Theorie für sich genommen jeweils Vor- und Nachteile. Für die In-plane-Technik spricht etwa die gleichzeitige Darstellbarkeit von Spitze und Nadelkörper, was eine bessere anatomische Orientierung ermöglichen kann. Die Out-of-plane-Technik hingegen könnte eine bessere Anlagemöglichkeit bieten, da die Nadel in anatomischer Laufrichtung des Nerven geführt wird (Fredrickson und Danesh-Clough 2013). Aus diesen Gegebenheiten lässt sich postulieren, dass eine Anlage in der In-plane-Technik zu mehr Luxationen bzw. Fehllagen des Katheters führen könnte, da dieser über einen $90^{\circ}$-Winkel zum Nerv eingeführt werden muss. Zum Vergleich dieser beiden Ultraschalltechniken existiert bislang nur eine Untersuchung zur interscalinären Katheteranlage bei Schulteroperationen (Fredrickson et al. 2011) und zur Nervus-femoralis-Katheteranlage bei Knieoperationen (Fredrickson und Danesh-Clough 2013), die sich mit der Qualität der postoperativen Analgesie befassen. Diese Studien konnten jedoch bislang keinen eindeutigen Vorteil für eines der beiden Verfahren herausarbeiten. Eine weitere Studie von Ruiz et al. beschäftigt sich mit der Wahrscheinlichkeit einer intraneuralen Punktion je nach Anlagetechnik. Hier zeigte sich ebenfalls ein leichter Vorteil für die In-plane Technik. Unter Verwendung der IP-Technik sind die Nadel und die Zielstruktur jederzeit einsehbar, wohingegen bei Verwendung der OOPTechnik die Nadel zunächst blind vorgeschoben werden muss. Eine akzidentelle Punktion des Nervs ist deshalb wahrscheinlicher (Ruiz et al. 2014). 


\subsection{Proximale Femurfrakturen}

Gegenstand dieser Arbeit ist die anästhesiologische Behandlung von Patienten mit einer Schenkelhalsfraktur mittels einer perioperativen Nervus-femoralis-Blockade.

Besondere Relevanz erhält dieses Thema durch die eminente Problematik und die hohen Fallzahlen der Schenkelhalsfrakturen bei geriatrischen Patienten. So gab es allein im Jahr 2004 Schätzungen zufolge 116281 Patienten mit mindestens einem Krankenhausaufenthalt wegen einer Hüftfraktur; dies entspricht einer Inzidenz von 140,9 pro 100.000 Einwohnern (Icks et al. 2008). In den Industrienationen wird die Inzidenz mit 150-250/100.000 angegeben (Kanis et al. 2012). Mit einer Verschärfung der Problematik ist aufgrund des demographischen Wandels und der damit einhergehenden zu erwartenden steigenden Inzidenz dieser Frakturen zu rechnen (Konnopka et al. 2009). Zahlen aus England beziffern die postoperative 30-Tage-Mortalitätsrate für Femurfrakturen mit 5-10\% (diese Zahl blieb in den letzten 20 Jahren unverändert) (Foss 2004).

Die große Masse der Patienten mit einer proximalen Femurfraktur bildet ein recht homogenes Patientenkollektiv. Laut einer Studie des NHS Hip Fracture Anaesthesia Network beträgt das durchschnittliche Alter dieser Patienten 81 Jahre, und es handelt sich dabei zu 73\% um Frauen. Generell nimmt die Inzidenz von proximalen Femurfrakturen mit steigendem Lebensalter deutlich zu. Postmenopausale Frauen sind am häufigsten von Osteoporose betroffen. Im Alter von 55 Jahren liegt die Prävalenz bereit bei 7\% und steigt auf 19\% bei 80-jährigen Frauen an (Leitlinie Osteoporose 2017). Weiterhin weist die Mehrzahl dieser Patienten, im Durchschnitt mit einer ASA-Stufe III, signifikante Co-Morbiditäten auf (White et al. 2010).

Femurfrakturen stellen eine besonders schmerzhafte Knochenverletzung dar, da das Periost hier eine besonders geringe Schmerzschwelle aufweist (Duc et al. 1994). Daten einer Metaanalyse aus dem Jahre 2010 weisen darauf hin, dass das perioperative Komplikationsrisiko wie etwa durch Delire, Pneumonien, Beinvenenthrombosen und pulmonale Embolien durch den Einsatz von Regionalanästhesieverfahren gesenkt werden kann (Luger et al. 2010). Ob sich hierdurch ein positiver Einfluss auf die Mortalität bei Patienten mit Femurfrakturen ergibt, konnte bisher nicht nachgewiesen werden (Parker et al. 2001; Luger et al. 2010).

Proximale Femurfrakturen beschreiben Schenkelhalsfrakturen sowie per- und subtrochantäre Femurfrakturen. Als Einteilung für Schenkelhalsfrakturen dient sowohl die PauwelsKlassifikation als auch die Klassifikation nach Garden. In der Klassifikation nach Pauwels 
werden drei Frakturtypen unterschieden. Die Typisierung richtet sich nach dem Winkel der Frakturlinie im Verhältnis zu einer Horizontalen (Pauwels I-III).

Die Gardenklassifikation richtet sich nach dem Dislokationsgrad der Fraktur. Hierbei werden Typ I-IV voneinander unterschieden. Anhand dieser Einteilung ist auch das Risiko einer gestörten Blutversorgung des Femurkopfs abschätzbar, dass mit einer zunehmenden Dislokation einhergeht. Somit ist die Gardenklassifikation ein wichtiger Parameter in der Entscheidung, ob eine gelenkerhaltende Therapie möglich ist oder ob ein kompletter Gelenkersatz stattfinden muss.

Pertrochantäre Femurfrakturen werden nach der AO-Klassifikation eingeteilt. Hier wird jeweils in drei Frakturtypen unterschieden. Typ A1 bezeichnet eine einfache Fraktur mit medialer Abstützung, Typ A2 eine mehrfragmentäre Fraktur unter Beteiligung des Trochanters minor ohne medialer Abstützung, und Typ A3 eine intertrochantäre Fraktur.

Subtrochantäre Femurfrakturen werden nach der AO-Klassifikation für lange Röhrenknochen eingeteilt (Arbeitsgemeinschaft für Osteosynthesefragen 2010). Für die Versorgung der Schenkelhalsfrakturen stehen sowohl osteosynthetische Verfahren als auch endoprothetische und konservative Maßnahmen zur Verfügung. Eine osteosynthetische, also gelenkerhaltende Therapie bei medialen Schenkelhalsfrakturen erfolgt meist über den Einsatz einer dynamischen Hüftschraube (DHS). Für endoprothetische Maßnahmen stehen Hemiprothesen (zumeist Duokopfprothesen) und Totalendoprothesen (TEP) zur Verfügung.

Stabile pertrochantäre Femurfrakturen können ebenfalls mit einer DHS versorgt werden, bei Instabilität muss auf einen Marknagel zurückgegriffen werden. Der Marknagel dient ebenfalls zur Versorgung der subtrochantären Frakturen (Hack et al. 2015). 


\section{$2 \quad$ Material und Methoden}

Diese prospektiv randomisierte Studie erhielt ein positives Votum (Antrag: 11/5/14) von der Ethikkommission der Georg-August-Universität Göttingen am 20.01.2015 und ist im Deutschen Register für Klinische Studien (DRKS: 00011585) am 18.1.17 registriert worden.

\subsection{Rekrutierung der Patienten}

In die Studie eingeschlossen wurden Patienten mit einer ASA-Klassifikation I-III im Alter von 18 bis 100 Jahren, die sich einer hüftgelenksnahen Operation (totale Endoprothese (Hüft-Tep), dynamischer Hüftschraube (DHS) oder intramedulärem Marknagel (PFN)) unterzogen und keine Kontraindikationen für eine kontinuierliche Nervus-femoralisBlockade aufwiesen. Die Rekrutierung der Patienten fand vom August 2016 bis zum Ende Januar 2017 statt. Im Rahmen der perioperativen Evaluation der Patienten wurde das Schmerzniveau mittels der NRS sowohl in Ruhe als auch bei Bewegung, perioperativer Analgetikaverbrauch, vorbestehende Parästhesien an der zu operierenden Extremität und allgemeine Patientendaten erfragt (s. Anhang 6.1). Nachdem die Patienten in die Operation, das Narkoseverfahren und die Teilnahme an der Studie eingewilligt hatten (s. Anhang 6.2), wurden sie mittels eines online Randomisierungsprogramms (Research Randomizer $\mathrm{C}$, Urbaniak GC, Plous S) entweder der In-plane-Gruppe oder der Out-of-plane-Gruppe zugeteilt.

\subsubsection{Anlage des Nervus-femoralis-Katheters}

Die Patienten wurden in üblicher Weise auf die Operation und Anästhesie vorbereitet: Blutdruckmanschette, Pulsoximeter, EKG-Elektroden und ein peripher venöser Zugang wurden angebracht. Zur Prämedikation erhielten die Patienten 60 Minuten vor der geplanten Operation gewichtsadaptiert 3,75-7,5 mg Midazolam p.o. Die Anlage des Nervusfemoralis-Katheters erfolgte präoperativ im Bett, um bereits vor Umlagerung des Patienten auf den Op-Tisch eine entsprechend gute Analgesie zu ermöglichen. War dies aufgrund der Nähe zum OP-Gebiet aus Sterilitätsgründen nicht möglich, erfolgte die Anlage direkt postoperativ.

Mit dem Ultraschallgerät (12 MHz Schallkopf, M-Turbo, FUJIFILM SonoSite, Bothell, USA) wurde im Bereich der Leistenfalte der Nervus femoralis aufgesucht und die Femoralarterie, die Femoralvene und der Nervus femoralis in der kurzen Achse dargestellt. Nach 
Desinfektion und steriler Abdeckung der Leistengegend und des Ultraschallkopfes wurde die geplante Punktionsstelle mit $2 \mathrm{ml}$ Mepivacain 1\% lokal anästhesiert. Unter Ultraschallsicht wurde mit einer Punktionsnadel der Nervus femoralis aufgesucht. Dies geschah, je nach Studiengruppe, entweder in der In-plane- oder in der Out-of-plane- Technik. Für die Punktion mittels Out-of-plane Technik wurde eine Facettenschliffnadel (SonoLong Nanoline; 19Gx 50mm; Pajunk GmbH, Geisingen, Deutschland) gewählt (s. Abbildung 6).

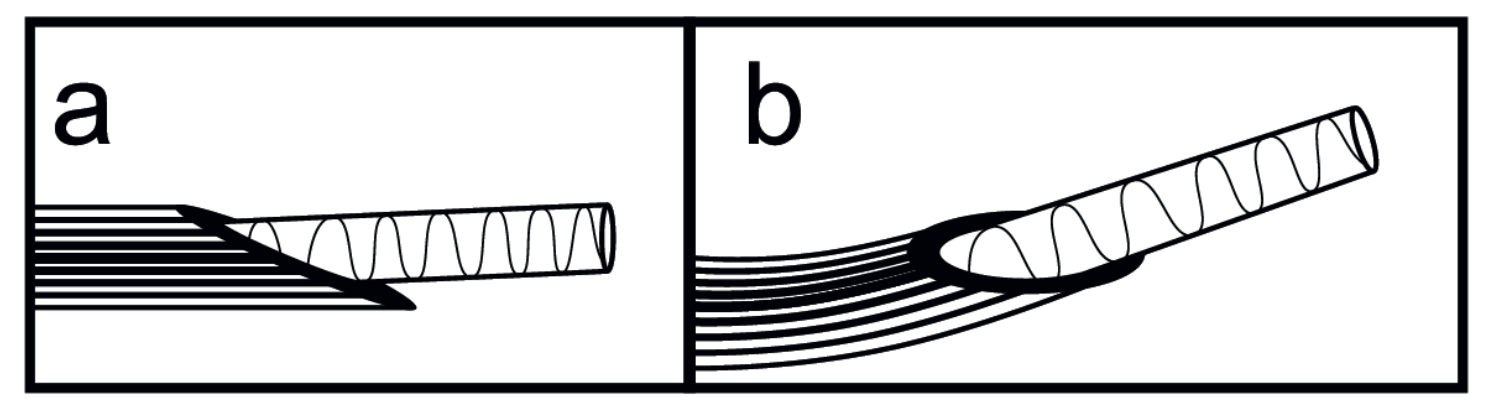

Abbildung 6: Nadelschliff (a) Facettenschliff, (b) Tuohy-Nadel

Die Punktion erfolgte ca. $3 \mathrm{~cm}$ distal des Ultraschallkopfes. Die Nadel wurde in einem $45^{\circ}$ -Winkel mittels der „Walk down Technik“ eingeführt (Tsui und Dillane 2006). Es erfolgten mehrere Aspirationsversuche, um eine akzidentielle Gefäßpunktion auszuschließen. Nach sicherer Darstellung des Nervs wurden zunächst $10 \mathrm{ml}$ Ropivacain $0,75 \%$ sowie $10 \mathrm{ml}$ Prilocain 1\% unter Ultraschallkontrolle durch die liegende Nadel verabreicht, bis der Nerv in Gänze von Lokalanästhetikum umgeben war. Anschließend wurde der Katheter (SonoLong Nano Line; 18Gx 50 mm Sono Katheter; 20Gx 50 cm; Pajunk GmbH, Geisingen, Deutschland) über die Nadel eingebracht. Die Spitze des Katheters wurde so einen bis sechs Zentimeter über die Nadelspitze hinaus, je nachdem ob ein signifikanter Gewebswiederstand auftrat, entlang des Nervs vorgeschoben (s. Abbildung 7). 


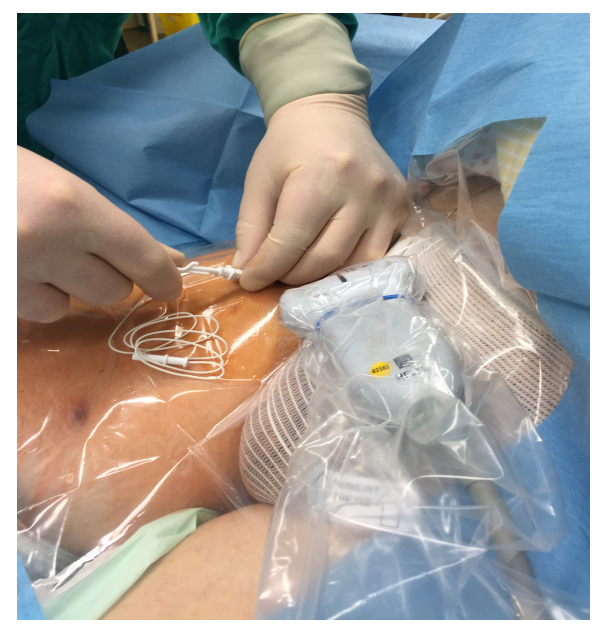

Abbildung 7: Anlage des Nervenkatheters

Abschließend wurden einige Milliliter Natriumchlorid über den liegenden Katheter verabreicht, um mittels Farbduplexsonographie über das entstehende visuelle Signal die korrekte Lage der Katheterspitze zu verifizieren. Analog zu dieser Technik wurde für die Anlage der In-plane-Katheter eine 18 G Tuohy-Nadel (SonoLong; Nano Line; Pajunk GmbH, Geisingen, Deutschland) verwendet (s. Abbildung 6). Die Punktion erfolgte hier ca. einen Zentimeter lateral zum Ultraschallkopf.

Während der Injektion wurden die Patienten engmaschig überwacht. Anschließend erfolgte die Annaht, und ein steriler Verband auf die Punktionsstelle wurde angelegt. Nach Feststellung der Wirksamkeit der regionalen Blockade durch sensorische und motorische Testung wurden die Patienten für die Operation freigegeben und mit der Allgemeinanästhesie bzw. mit der Spinalanästhesie begonnen. Zirka dreißig Minuten vor dem Operationsende erhielten die Patienten ohne Spinalanästhesie eine gewichtsadaptierte Einmalgabe Metamizol i. V. Die Dokumentation der Katheteranlage erfolgte im Anlageprotokoll (s. Anhang 6.3).

Postoperativ wurden die Katheter an Schmerzpumpen angeschlossen (PEGA PCA; Venner Medical; Dänischhagen, Deutschland). Die Schmerzpumpen verfügten sowohl über eine basale Laufrate als auch über eine Bolusfunktion, die vom Patienten selbst ausgelöst werden konnte. Der Patient erhielt initial eine basale Laufrate von $5 \mathrm{ml} / \mathrm{h}$ Ropivacain $0,2 \%$, bei Schmerzen konnten die Patienten $5 \mathrm{ml}$ Boli mit einer Sperrzeit von 30min abfordern. Im Rahmen der täglichen Schmerzvisite wurde die basale Laufrate der Pumpen bedarfsgerecht nachjustiert. Bei einem NRS> 3 erhielten die Patienten im Aufwachraum zusätzliche Gaben von Oxycodon i. V. 


\subsubsection{Allgemeinanästhesie}

Die Allgemeinanästhesie wurde in der totalintravenösen Technik (TIVA) durchgeführt. Hierbei wurden Sufentanil und Propofol zum Einsatz gebracht. Die Anästhesie wurde begonnen durch die Verabreichung eines Sufentanil-Bolus $(0,3 \mu \mathrm{g} / \mathrm{kgKG})$, gefolgt von einer Bolusinjektion von Propofol ( $2 \mathrm{mg} / \mathrm{kgKG}$ über zwei Minuten). Nach Relaxierung mit Rocuronium $(0,6 \mathrm{mg} / \mathrm{kgKG})$ wurde der Patient, bei ausreichender Narkosetiefe, intratracheal intubiert. Die initiale Beatmung des Patienten wurde mit einer 40 prozentigen Sauerstoffkonzentration und einem Tidalvolumen von initial $7 \mathrm{ml} / \mathrm{kgKG}$ und einer Frequenz von 15/min begonnen. Diese Einstellungen wurden im Verlauf an das endexpiratorische $\mathrm{CO} 2$ angepasst, so dass dieses zwischen 35 und $45 \mathrm{mmHG}$ lag. Die Aufrechterhaltung der Narkose erfolgte durch eine kontinuierliche Gabe von Propofol über einen Perfusor mit initial $4 \mathrm{mg} / \mathrm{kg} \mathrm{KG} / \mathrm{h}$ und repetetiven Sufentanilboli $(0.1 \mu \mathrm{g} / \mathrm{kg} \mathrm{KG}$ ). Die Rate der Propofolinfusion und die Menge des verabreichten Sufentanil wurde so gewählt, dass der mittlere arterielle Druck (MAD) zwischen 60 und $100 \mathrm{mmHG}$ und die Herzfrequenz zwischen 50 und 100/min lag. Die Extubation des Patienten erfolgte, nachdem der Patient über ausreichende Schutzreflexe und eine Atemfrequenz von $>10 / \mathrm{min}$ verfügte. Dies war zumeist während oder kurz nach der Anlage des Verbandes der Fall.

\subsubsection{Spinalanästhesie}

Die Durchführung der Spinalanästhesie erfolgte in der Regel am sitzenden Patienten. War das Sitzen aufgrund von Mobilitätseinschränkung oder Schmerzen nicht möglich, so wurde der Patient gebeten sich auf die Seite zu legen, so dass die zu operierende Hüfte nach oben zeigte. Der L4 Dornfortsatz wurde durch Palpation, in der Mitte einer gedachten Linie zwischen den Crista iliaca superior, identifiziert. Anschließend wurde eine lokale Betäubung der Haut mit Mecain 1\% durchgeführt. Zunächst wurde eine 26 G Spinalnadel (Atraucan, B. Braun Melsungen, Melsungen Deutschland) in den Zwischenraum L4/L5 eingeführt. War dies aufgrund schlechter anatomischer Voraussetzungen nicht möglich, so erfolgte eine zweite Punktion in Höhe L3/L4. Sobald der Spinalkanal erreicht wurde und Liquor im Konus der Spinalnadel erschien, wurde 0,5\% isobares Bupivacain (Carbostesin, AstraZeneca, London, United Kingdom) appliziert. Die regelrechte Ausbreitung der Spinalanästhesie wurde nach ca. 10-15 min mittels eines Kältesprays überprüft und der Patient zur Operation freigegeben. Eine adäquate Rückbildung der Spinalanästhesie wurde postoperativ durch das Personal des Aufwachraumes überprüft. 


\subsubsection{Schmerzpumpen und Schmerzvisite}

Im Aufwachraum wurden die Nervenkatheter an eine mobile Infusionspumpe angeschlossen (PEGA PCA; Venner Medical; Dänischhagen, Deutschland), über die kontinuierlich Ropivacain 0,2\% (initial $5 \mathrm{ml} / \mathrm{h}$ ) injiziert wurde. Bei Bedarf konnten sich die Patienten halbstündlich einen Bolus ( $5 \mathrm{ml}$ Ropivacain 0,2\%) über einen Druckknopf selbst verabreichen. Patienten mit einer Schmerzintensität $>3$ auf der Numerischen Rating Skala (NRS) bekamen zusätzlich $1 \mathrm{mg}$ Oxycodon intravenös verabreicht. Die Patienten wurden an den Folgetagen täglich von einer Pain Nurse visitiert. Bei weiterhin bestehender Schmerzsymptomatik (NRS > 3) wurde die Laufrate der Infusionspumpe in $2 \mathrm{ml}$ Schritten erhöht. Für die ersten zwei postoperativen Tage erhielten die Patienten zusätzlich ein festes analgetisches Regime, bestehend aus einer oralen Gabe von drei mal 600mg Ibuprofen und zwei mal 10/5mg Targin pro Tag.

\subsubsection{Studienprotokoll und Datenerhebung}

Präoperativ wurden die Patienten wie üblich im Rahmen der Narkoseaufklärung visitiert. Im Rahmen dieser präoperativen Visite wurden die Patienten über mögliche Narkose- und Katheterverfahren sowie über deren jeweilige Risiken und Nebenwirkungen aufgeklärt. Kam für die Patienten ein Nervus-femoralis-Katheter in Frage und waren die Patienten damit einverstanden, erfolgt eine zweite Visite. In der zweiten Visite wurden die Patienten über den Studienablauf informiert und um eine Einverständniserklärung zur Studie gebeten (s. Anhang 6.2). Im Rahmen dieses Gesprächs werden außerdem allgemeine Daten erhoben, hierzu zählen: Alter, Geschlecht, Größe, Gewicht, Diagnose(n), Art der operativen Versorgung, ASA-Klassifikation, vorbestehende chronische Schmerzen sowie der Gebrauch von Schmerzmitteln (s. Anhang 6.1).

Des Weiteren wurde eine Schmerzanamnese erhoben. Der Schmerzgrad der Patienten wurde anhand der numerischen Rating-Skala belegt (s. Abbildung 8).

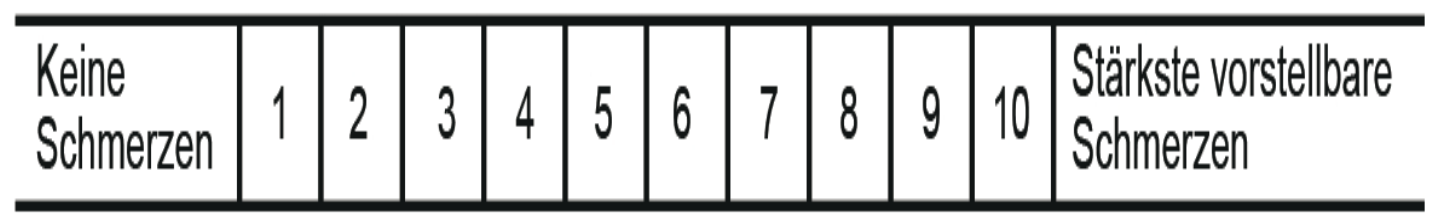

Abbildung 8: Numerische Rating-Skala Schmerz

Die Patienten wurden anhand der NRS gebeten, den Schmerzgrad in Ruhe und bei Bewegung zu beziffern. 
Die Muskelkraft der zu operierenden Extremität wurde anhand des Kraftgrades nach Janda angegeben. Hierbei handelt es sich um eine semiquantitative Methode zur Abschätzung der Muskelkraft; in Anlehnung an das Britisch Medical Research Council wird sie in 6 Kraftgrade eingeteilt (0-5):

Tabelle 1: Kraftgrad nach Janda

\begin{tabular}{|c|c|c|c|c|c|}
\hline 0 & 1 & 2 & 3 & 4 & 5 \\
\hline $\begin{array}{l}\text { Keine mus- } \\
\text { kuläre Akti- } \\
\text { vität vor- } \\
\text { handen }\end{array}$ & $\begin{array}{l}\text { Sichtbare und } \\
\text { oder tastbare } \\
\text { Kontraktionen } \\
\text { ohne Bewe- } \\
\text { gung }\end{array}$ & $\begin{array}{l}\text { Bewegungen } \\
\text { unter Aus- } \\
\text { schaltung der } \\
\text { Schwerkraft } \\
\text { möglich }\end{array}$ & $\begin{array}{l}\text { Bewegungen } \\
\text { gegen die } \\
\text { Schwerkraft } \\
\text { möglich }\end{array}$ & $\begin{array}{l}\text { Bewegungen } \\
\text { gegen leich- } \\
\text { ten Wider- } \\
\text { stand }\end{array}$ & $\begin{array}{l}\text { Normale } \\
\text { Muskelkraft }\end{array}$ \\
\hline
\end{tabular}

Im Zusammenhang mit den Kraftgraden erfolgte auch eine Erfassung des Grades der Mobilisation des Patienten. Die Mobilisierbarkeit wurde anhand von 4 Graden klassifiziert und dokumentiert:

Tabelle 2: Mobilisationsgrade

\begin{tabular}{|l|l|l|l|}
\hline 0 & 1 & 2 & 3 \\
\hline Liegt & Sitzt & $\begin{array}{l}\text { Eingeschränktes } \\
\text { Gehen }\end{array}$ & Uneingeschränkt \\
\hline
\end{tabular}

Diese Parameter wurden nochmals am OP-Tag sowie am ersten und zweiten postoperativen Tag abgefragt. Zusätzlich wurden die Patienten gebeten, unter laufender Analgesie mit dem Nervus-femoralis-Katheter die sensorische Sensibilität in den Gebieten der Rami cutanei anteriores des Nervus femoralis (anteriorer Oberschenkel), des Nervus cutaneus femoris lateralis (lateraler Oberschenkel) und des Nervus obturatorius (kleine Aussparung am medialen Oberschenkel) anzugeben. Die Einschätzung der Parästhesie erfolgt täglich. Die Patienten wurden hierbei gebeten, den Grad der Parästhesie im jeweiligen Gebiet anzugeben. Die Einschätzung der Parästhesie erfolgte anhand von 3 Graden: 
Tabelle 3: Einschätzung von Parästhesien

\begin{tabular}{|l|l|l|}
\hline 0 & 1 & 2 \\
\hline Keine Parästhesie im be- & Vermindertes Kälte- & Ausgeschaltetes Kälte- \\
troffenen Gebiet & empfinden und leichte & $\begin{array}{l}\text { empfinden und komplettes } \\
\text { Taubheitsgefühl }\end{array}$ \\
& Taubheit & \\
\hline
\end{tabular}

An allen drei Tagen wurde auch die Patientenzufriedenheit auf einer Skala von 1-10 (analog zur NRS) abgefragt. Laufrate und Abfrage gebrauchter zusätzlicher Boli durch die Schmerzpumpe wurden jeweils im Rahmen der morgendlichen Schmerzvisite erfasst (s. Anhang 6.4).

\subsubsection{Das postoperative Röntgenbild}

Die radiologische Kontrolle der versorgten Hüfte/Femur erfolgt normalerweise am zweiten postoperativen Tag nach Entfernung der eingebrachten Wunddrainagen. Ist am zweiten postoperativen Tag noch eine Zunahme des Blutvolumens in der Drainage festzustellen, wird die Drainage vorerst noch belassen. Zunächst erfolgt eine A.P. (Anterior/Posterior)- Aufnahme des Beckens (Beckenübersicht). Der Patient befindet sich in Rückenlagerung, die Beine sind leicht gespreizt und die Fußspitzen nach innen rotiert. Hierbei stellt sich das Becken vollständig symmetrisch dar, beide Hüftgelenke und die Trochanteren sind einsehbar. Des Weiteren erfolgt eine A.P. -Einzelaufnahme des operierten Hüftgelenkes. Nach einer Versorgung mit einer totalen Endoprothese erfolgt außerdem eine axiale Hüftaufnahme nach Lauenstein. Hierbei befindet sich der Patient ebenfalls in Rückenlage, das zu untersuchende Bein wird so stark gebeugt, bis die Fußsohle flach auf dem Tisch steht. Der Femur steht nun fast senkrecht zum Tisch und wird dann $15^{\circ}$ nach außen gekippt, um den Schenkelhals zu untersuchen, oder er wird stark nach außen in rund $45^{\circ}$ gekippt, um den Hüftgelenkkopf zu untersuchen. Hierbei ist eine gute Beurteilung des Hüftkopfes, der Pfanne und des Schenkelhalses möglich. Zur genauen Feststellung der Katheterlage und zur genaueren Analyse erfolgte die Auswertung der Röntgenbilder unter Mithilfe des radiologischen Oberarztes Dr. med. Alexander Schwarz. 


\subsubsection{Definierung des Zielgebietes}

Um eine standardisierte Beurteilung des Katheterverlaufs und insbesondere der Katheterspitze vornehmen zu können, wurden für die A.P.-Röntgenaufnahmen eine Zielregion (Region 0) und vier weitere angrenzende Regionen (Regionen 1a, 1b, 2, 3) definiert. Die Zielregion (Region 0) projiziert sich hierbei in den Röntgenaufnahmen über dem Hüftkopf, welcher an dieser Stelle von dem Stamm des Nervus femoralis gekreuzt wird. In diesem Bereich verläuft der Nerv auf dem Musculus iliacus und lateral des Musculus psoas major. Unter dem Ligamentum inguinale vereinigen sich diese beiden Muskeln und ziehen über das Hüftgelenk zum Trochanter minor des Femurs. Linie 1 verläuft proximal des Trochanters minor in das Zentrum der Zielregion (=Hüftkopf). Im Zentrum der Zielregion schneidet Linie 1 eine Linie 2, welche parallel zum Ligamentum inguinale angeordnet ist (s. Abbildung 9). Linie 1 und Linie 2 teilen die Zielregion in die folgenden vier Quadranten: proximal-lateral (1a) und proximal-medial (1b) nach der Gruppe 3 und der Gruppe 2 definiert durch Capdevila et al. (Capdevila et al. 2002), sowie distal-lateral (2) und distal-medial (3) (s. Abbildung 9).

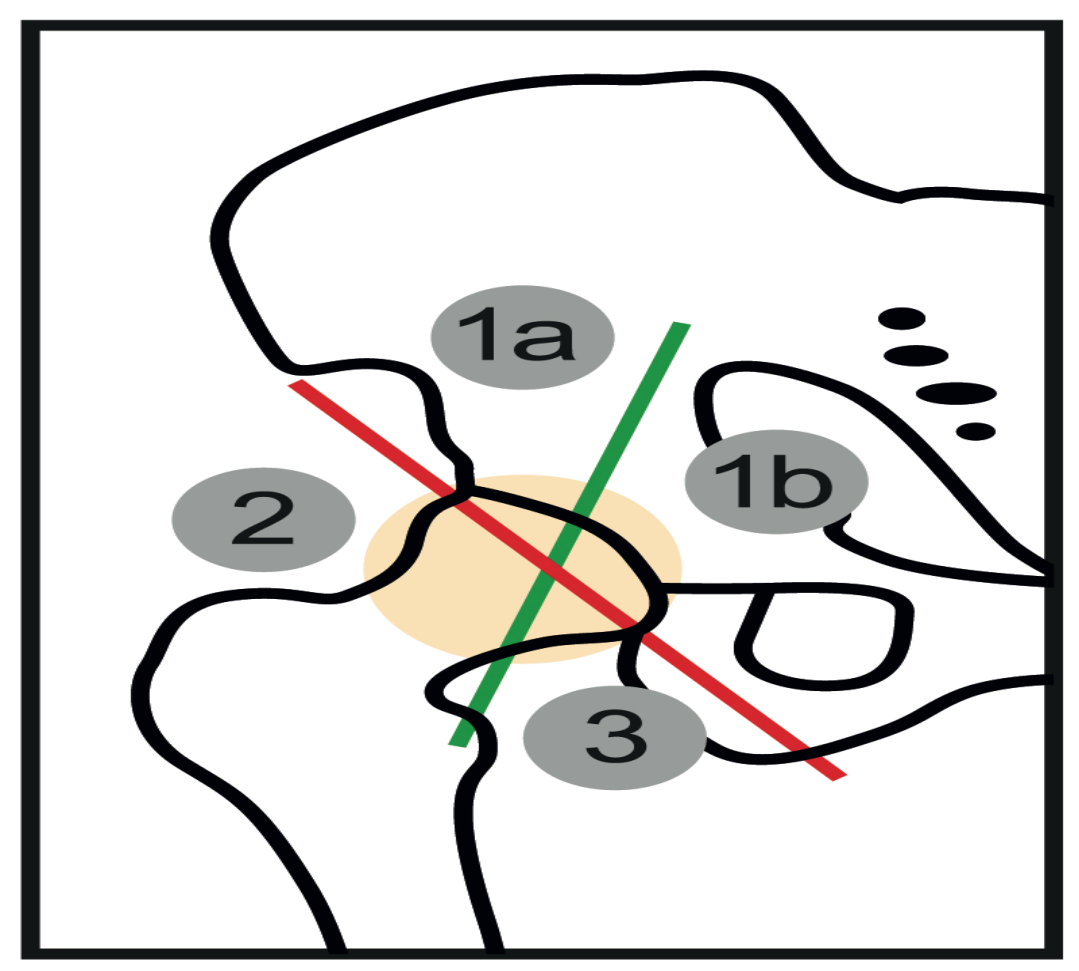

Abbildung 9: Zielgebiet, Linie 1 (grün), Linie 2 (rot), Zielgebiet (gelb)

Im Falle einer implantierten Endoprothese wurden die Umrisse des Kopfes der Prothese äquivalent zum Hüftkopf als Zielregion gewertet. Konnte die Katheterspitze aufgrund einer Überschattung durch die Hüftprothese nicht einwandfrei zugeordnet werden, wurde eine axiale Röntgenaufnahme zur besseren Beurteilbarkeit herangezogen. Die Katheter 
wurden als nach „distal umgeschlagen“ gewertet, wenn die Ausrichtung des 4,5 cm langen Katheterendes von Linie $1>90^{\circ}$ abwich.

\subsubsection{Statistische Auswertung}

Der primäre Endpunkt der Studie wurde durch die Lokalisation der Katheterspitze innerhalb der Zielregion (Ja/Nein) definiert. Sekundärer Endpunkt der Studie war die Entfernung der Katheterspitze zur Zielregion und ihre Orientierung in den angrenzenden Regionen. Weitere sekundäre Endpunkte der Studie umfassten das Schmerzempfinden, die Patientenzufriedenheit, den Verbrauch von Lokalanästhetika via Infusionspumpen, Sensibilitätsverlust und Muskelkraft in der operierten Extremität sowie den postoperativen Grad der Mobilisierung. Die statistische Auswertung erfolgte mittels des StatSoft (Dell Inc., Texas, USA) Programms sowie Microsoft Office Excel 2011 (Microsoft Corporation, Redmond, USA). Die Testung der Daten auf Normalverteilung erfolgte mit dem Kolmogorov-Smirnov-Test. Wurde eine Normalverteilung angenommen, wurden ordinalskalierte Daten als Mittelwert und Standardabweichung angegeben. Kategoriale Daten wurden als prozentuale Anteile angegeben. Die Testung auf Unterschiede zwischen den Gruppen erfolgte für ordinalskalierte Daten mit dem Student-t-Test. Ein p-Wert kleiner als 0,05 wurde als statistisch signifikant erachtet. 


\section{Ergebnisse}

In dieser Studie wurden 34 Patienten untersucht und in die Studie aufgenommen.

Bei 16 Patienten wurde ein Nervus-femoralis-Katheter mittels In-plane-Technik angelegt (IP-Gruppe). Bei weiteren 18 Patienten wurde ein Katheter mittels Out-of-plane-Technik angelegt (OOP-Gruppe). In Bezug auf die allgemeinen Patientendaten wie Alter, Geschlecht, Größe, Gewicht, ASA- und NYHA-Klassifikation waren beide Gruppen vergleichbar (s. Tabelle 4).

\subsection{Allgemeine Patientendaten}

In der In-Plane Gruppe erhielten 15 Patienten eine Hüft-TEP sowie zwei weitere Patienten einen pertrochantären Femurnagel. Vergleichbares gilt für die Out-of-plane-Gruppe mit 16 Hüft-TEPs, einem PFN und einer DHS. Davon wurden in der IP-Gruppe sieben dringlich durchgeführt und neun unter elektiven Rahmenbedingungen. Bei der OOP-Gruppe waren es 6 dringlich und 12 elektiv durchgeführte Operationen. Es erhielten 11 Patienten der IPGruppe eine Spinalanästhesie und 10 in der OOP-Gruppe, eine Vollnarkose erhielten fünf Patienten der IP-Gruppe und acht Patienten der OOP-Gruppe.

Tabelle 4: Allgemeine Patientendaten

\begin{tabular}{cccc}
\hline & IP & OOP & $\mathrm{p}$ \\
& $\mathrm{n}=16$ & $\mathrm{n}=18$ & \\
\hline Alter (Jahren) & $69,0 \pm 13,4$ & $65,4 \pm 18,9$ & 0.53 \\
\hline Geschlecht (männlich/weiblich), & $7 / 9$ & $8 / 10$ & 0,97 \\
\hline Größe (cm) & $171,7 \pm 10,1$ & $170.0 \pm 7,5$ & 0.58 \\
\hline Gewicht (kg) & $78,8 \pm 9,4$ & $76,1 \pm 14,7$ & 0,53 \\
\hline SPA/VN, n & $11 / 5$ & $10 / 8$ & 0,43 \\
\hline Dringlich/ elektiv, n & $7 / 9$ & $6 / 12$ & 0,53 \\
\hline TEP/PFN/DHS, n & $15 / 2 / 0$ & $16 / 1 / 1$ & 0,47 \\
\hline
\end{tabular}




\begin{tabular}{rrrr}
\hline ASA (I/II/III), n & $0 / 10 / 6$ & $3 / 10 / 5$ & 0,22 \\
\hline NYHA (I/II/III), n & $11 / 4 / 0$ & $15 / 2 / 1$ & 0,36 \\
\hline
\end{tabular}

\subsection{Numerische Rating-Skala}

In der IP- sowie in der OOP-Gruppe gaben 16 Patienten an, präoperativ Schmerzen gehabt zu haben. In der OOP-Gruppe waren zudem zwei Patienten präoperativ schmerzfrei. In der IP-Gruppe bestand präoperativ ein Mittel von 1,6 $( \pm 1,2)$ auf der NRS in Ruhe sowie 5,4 ( $\pm 1,5)$ unter Bewegung. Es bestand ein leicht erhöhtes Schmerzniveau in der OOPGruppe mit 2,6 $( \pm 1,9)$ in Ruhe und 5,8 $( \pm 1,8)$ in Bewegung. Postoperativ gaben 9 Patienten der IP-Gruppe und 8 Patienten der OOP-Gruppe Schmerzen an $(\mathrm{p}=0.49)$. Die NRS der IP-Gruppe lag in Ruhe bei 1,4 ( $\pm 1,0)$ und bei 3,0 $( \pm 2,1)$ in Bewegung. Die postoperative NRS der OOP-Gruppe lag etwas höher bei 3,1 $( \pm 2,0)$ in Ruhe sowie bei 4,3 $( \pm 2,0)$ in Bewegung. Am ersten postoperativen Tag gaben 14 Patienten der IP-Gruppe Schmerzen an (2 schmerzfreie Patienten). In der OOP-Gruppe gaben alle 18 Patienten Schmerzen am ersten postoperativen Tag an. Das Schmerzempfinden war in der IP- Gruppe etwas niedriger mit $1,6( \pm 1,1)$ in Ruhe und 2,9 $( \pm 1,2)$ in Bewegung als in der OOP-Gruppe mit 2,3 $( \pm 5,2)$ in Ruhe und 3,6 ( $\pm 2,0)$ in Bewegung. Am zweiten postoperativen Tag gaben bereits vier Patienten der IP-Gruppe an, schmerzfrei zu sein (12 Patienten mit Schmerzen). In der OOPGruppe gaben weiterhin alle 18 Patienten Schmerzen an. Dabei zeigte sich auf der NRS in der IP-Gruppe 1,3 $( \pm 0,6)$ in Ruhe und 2,0 $( \pm 1,2)$ bei Bewegung zu 1,9 $( \pm 1,4)$ in Ruhe und $2,9( \pm 1,8)$ in Bewegung bei der OOP- Gruppe. 
Tabelle 5: Schmerzskala NRS

\begin{tabular}{|c|c|c|c|}
\hline & IP & OOP & $\mathrm{p}$ \\
\hline & $\mathrm{n}=16$ & $\mathrm{n}=18$ & \\
\hline NRS präoperativ in Ruhe & $1,6 \pm 1,2$ & $2,6 \pm 1,9$ & 0,05 \\
\hline $\begin{array}{c}\text { NRS präoperativ bei } \\
\text { Bewegung }\end{array}$ & $5,4 \pm 1,5$ & $5,8 \pm 1,8$ & 0.41 \\
\hline Schmerzen präoperativ, $n$ & $16(100 \%)$ & $16(88,9 \%)$ & 0,17 \\
\hline $\begin{array}{l}\text { Schmerzen postoperativ } \\
\qquad(\mathrm{ja} / \text { nein), } \mathrm{n}\end{array}$ & $9 / 7$ & $8 / 10$ & 0.49 \\
\hline NRS postoperativ in Ruhe & $1,4 \pm 1,0$ & $3,1 \pm 2,0$ & 0,04 \\
\hline $\begin{array}{c}\text { NRS postoperativ bei } \\
\text { Bewegung }\end{array}$ & $3,0 \pm 2,1$ & $4,3 \pm 2,0$ & 0.23 \\
\hline $\begin{array}{l}\text { Schmerzen am ersten } \\
\text { postoperativen Tag } \\
\text { (ja/nein), n }\end{array}$ & $14(87,5 \%)$ & $18(100 \%)$ & 0,12 \\
\hline $\begin{array}{c}\text { NRS am ersten } \\
\text { postoperativen Tag in } \\
\text { Ruhe }\end{array}$ & $1,6 \pm 1,1$ & $2,3 \pm 5.2$ & 0.16 \\
\hline $\begin{array}{c}\text { NRS am ersten } \\
\text { postoperativen Tag bei } \\
\text { Bewegung }\end{array}$ & $2,9 \pm 1,2$ & $3,6 \pm 2,0$ & 0,31 \\
\hline $\begin{array}{c}\text { Schmerzen am zweiten } \\
\text { postoperativen Tag } \\
\text { (ja/nein), n }\end{array}$ & $12(75 \%)$ & $18(100 \%)$ & 0,02 \\
\hline $\begin{array}{c}\text { NRS am zweiten } \\
\text { postoperativen Tag in } \\
\text { Ruhe }\end{array}$ & $1,3 \pm 0,6$ & $1,9 \pm 1,4$ & 0,01 \\
\hline $\begin{array}{c}\text { NRS am zweiten } \\
\text { postoperativen Tag bei } \\
\text { Bewegung }\end{array}$ & $2,0 \pm 1,2$ & $2,9 \pm 1,8$ & 0,15 \\
\hline
\end{tabular}




\subsection{Patientenzufriedenheit}

Postoperativ lag die Patientenzufriedenheit in der IP-Gruppe mit 8,5 $( \pm 1,2)$ geringfügig höher als in der OOP-Gruppe mit 8,1 $( \pm 2,3)$. Am ersten Tag lag die Patientenzufriedenheit in der IP-Gruppe mit 9,3 $( \pm 1,0)$ um 1,7 Punkte höher $(\mathrm{p}=0,02)$ als in der OOP-Gruppe mit 7,6 ( $\pm 2,6)$. Am zweiten postoperativen Tag gaben die Patienten der IP-Gruppe durchschnittlich eine Zufriedenheit von 9,5 ( $\pm 0,9)$, die Patienten der OOP-Gruppe durchschnittlich von 8,4 $( \pm 1,8)$ an. Damit lag auch am zweiten postoperativen Tag die Patientenzufriedenheit in der IP-Gruppe höher (durchschnittlich 1,1, p=0,03).

Tabelle 6: Patientenzufriedenheit

\begin{tabular}{cccc}
\hline & IP & OOP & $\mathrm{p}$ \\
& $\mathrm{n}=16$ & $\mathrm{n}=18$ & \\
\hline $\begin{array}{c}\text { Patientenzufriedenheit postoperativ }(1- \\
10)\end{array}$ & $8,5 \pm 1,2$ & $8,1 \pm 2,3$ & 0,5 \\
\hline $\begin{array}{c}\text { Patientenzufriedenheit am ersten } \\
\text { postoperativen Tag (1-10) }\end{array}$ & $9,3 \pm 1,0$ & $7,6 \pm 2,6$ & 0,02 \\
\hline $\begin{array}{c}\text { Patientenzufriedenheit am zweiten } \\
\text { postoperativen Tag (1-10) }\end{array}$ & $9,5 \pm 0,9$ & $8,4 \pm 1,8$ & 0,03 \\
\hline
\end{tabular}

\subsection{Schmerzpumpen}

Die basale Laufrate der Schmerzpumpen betrug in der IP-Gruppe unmittelbar postoperativ $6,4( \pm 1,2)$ sowie $5,8( \pm 1,0)$ in der OOP-Gruppe. Am ersten postoperativen Tag betrug die durchschnittliche Laufrate in der IP-Gruppe 5,8 $(2,0)$ und 6,0 $( \pm 1,0)$ in der OOP-Gruppe. Auch die Anzahl der vom Patienten abgerufenen Boli zeigte sich nahezu identisch bei 5,5 $( \pm 4,2)$ in der IP-Gruppe und 5,2 $( \pm 7,0 ; \mathrm{p}=0,85)$ in der OOP-Gruppe. Am zweiten postoperativen Tag lag die basale Laufrate der IP-Gruppe bei 4,0 $( \pm 3,5)$ und bei 3,7 $( \pm 3,2)$ in der OOP-Gruppe. Es wurden durchschnittlich 3,5 ( $\pm 4,5)$ Boli in der IP-Gruppe und 2,2 $( \pm 3,6)$ in der OOP-Gruppe abgerufen. 
Tabelle 7: Schmerzpumpen

\begin{tabular}{llll}
\hline & $\begin{array}{l}\mathrm{IP} \\
\mathrm{n}=16\end{array}$ & $\begin{array}{l}\text { OOP } \\
\mathrm{n}=18\end{array}$ & $\mathrm{P}$ \\
\hline PCA basale Laufrate postoperativ (1-10) & $6,4 \pm 1,2$ & $5,8 \pm 1,0$ & 0,14 \\
\hline PCA Boli abgerufen postoperativ, $\mathrm{n}$ & $0 \pm 0$ & $0,3 \pm 0,7$ & 0,12 \\
\hline $\begin{array}{l}\text { PCA basale Laufrate am ersten } \\
\text { postoperativen Tag (1-10) }\end{array}$ & $5,8 \pm 2,0$ & $6,0 \pm 1,0$ & 0,73 \\
\hline $\begin{array}{l}\text { PCA Boli abgerufen am ersten } \\
\text { postoperativen Tag, } \mathrm{n}\end{array}$ & $5,5 \pm 4,2$ & $5,2 \pm 7,0$ & 0,89 \\
\hline $\begin{array}{l}\text { PCA basale Laufrate am zweiten } \\
\text { postoperativen Tag (1-10) }\end{array}$ & $4,0 \pm 3,5$ & $3,7 \pm 3,2$ & 0,81 \\
\hline $\begin{array}{l}\text { PCA Boli abgerufen am zweiten } \\
\text { postoperativen Tag, } \mathrm{n}\end{array}$ & $3,4 \pm 4,5$ & $2,2 \pm 3,6$ & 0,37 \\
\hline
\end{tabular}

\subsection{Taubheit im Wirkungsgebiet}

In beiden Gruppen gab es keinen Patienten, der im Vorfeld der Operation eine Taubheit im zu untersuchenden Gebiet angab. Postoperativ gaben in der IP-Gruppe für den lateralen Oberschenkel sechs Patienten eine Taubheit Grad II an, neun Patienten eine Taubheit Grad I und ein Patient keine Taubheit an. In der OOP-Gruppe gaben 11 Patienten eine Taubheit Grad II an, sechs Patienten eine Taubheit Grad I, und ein Patient gab an, keine Taubheit im lateralen Oberschenkel zu verspüren $(\mathrm{p}=0,38)$.

Eine Taubheit Grad II am anterioren Oberschenkel gaben postoperativ fünf Patienten der IP-Gruppe und 12 Patienten der OOP-Gruppe an. Eine Taubheit Grad I verspürten neun Patienten der IP-Gruppe und fünf Patienten der OOP-Gruppe am anterioren Oberschenkel. Zwei Patienten der IP-Gruppe und ein Patient der OOP-Gruppe verspürten direkt postoperativ keinerlei Taubheit im anterioren Oberschenkel $(p=0,12)$. Für das Innervationsgebiet des Nervus obturatorius gaben in der IP-Gruppe vier Patienten eine komplette, vier Patienten eine Taubheit Grad I und acht Patienten keinerlei Taubheit an. In der OOPGruppe gaben zwei Patienten eine Taubheit Grad II, neun Patienten eine Taubheit Grad I und sieben Patienten keinerlei Taubheit im Obturatoriusgebiet an $(p=0,28)$. Am ersten postoperativen Tag gaben sechs Patienten der OOP-Gruppe und ein Patient der IPGruppe eine Taubheit Grad II des lateralen Oberschenkels und je 11 Patienten beider Gruppen eine Taubheit Grad I an. Keine Taubheit im lateralen Oberschekel verspürten vier Patienten der IP-Gruppe und ein Patient der OOP-Gruppe am ersten postoperativen Tag $(p=0,07)$. Für das Gebiet des Nervus obturatorius gab lediglich noch ein Patient der 
OOP-Gruppe eine Taubheit zweiten Grades an und kein Patient der IP-Gruppe. Eine Taubheit Grad I verspürten nur noch drei Patienten der OOP-Gruppe und ein Patient der IP-Gruppe am ersten postoperativen Tag $(\mathrm{p}=0,38)$. Am zweiten postoperativen Tag gab je ein Patient beider Gruppen ein Taubheitsgefühl Grad II für den anterioren Oberschenkel an, 10 der OOP-Gruppe und acht der IP-Gruppe ein Taubheitsgefühl Grad I und je sieben Patienten beider Gruppen gaben an, keinerlei Taubheit in dem Gebiet zu verspüren $(\mathrm{p}=0,64)$. Im Innervationsgebiet des Nervus obturatorius gaben lediglich noch zwei Patienten der OOP-Gruppe am zweiten postoperativen Tag ein Taubheitsgefühl Grad I an. Die restlichen Patienten beider Gruppen verspürten zu diesem Zeitpunkt keine Taubheit mehr $(\mathrm{p}=0,17)$.

Tabelle 8: Taubheit im Wirkungsgebiet

\begin{tabular}{|c|c|c|c|}
\hline & IP & OOP & $\mathrm{p}$ \\
\hline & $\mathrm{n}=16$ & $\mathrm{n}=18$ & \\
\hline Taubheit postoperativ: lat. OS $(0 / 1 / 2), \mathrm{n}$ & $1 / 9 / 6$ & $1 / 6 / 11$ & 0,38 \\
\hline Taubheit postoperativ: ant. OS $(0 / 1 / 2), \mathrm{n}$ & $2 / 9 / 5$ & $1 / 5 / 12$ & 0,12 \\
\hline Taubheit postoperativ: $\mathrm{N}$. obturatorius $(0 / 1 / 2), \mathrm{n}$ & $8 / 4 / 4$ & $7 / 9 / 2$ & 0,28 \\
\hline $\begin{array}{l}\text { Taubheit am ersten postoperativen Tag: lat. OS } \\
(0 / 1 / 2), \mathrm{n}\end{array}$ & $4 / 11 / 1$ & $1 / 11 / 6$ & 0,07 \\
\hline $\begin{array}{l}\text { Taubheit am ersten postoperativen Tag: ant. OS } \\
(0 / 1 / 2), \mathrm{n}\end{array}$ & $3 / 12 / 1$ & $2 / 8 / 8$ & 0,04 \\
\hline $\begin{array}{l}\text { Taubheit am ersten postoperativen Tag: } \mathrm{N} \text {. } \\
\text { obturatorius }(0 / 1 / 2), \mathrm{n}\end{array}$ & $15 / 1 / 0$ & $14 / 3 / 1$ & 0,38 \\
\hline $\begin{array}{l}\text { Taubheit am zweiten postoperativen Tag: lat. OS } \\
(0 / 1 / 2), \mathrm{n}\end{array}$ & $7 / 8 / 1$ & $7 / 10 / 1$ & 0,95 \\
\hline $\begin{array}{l}\text { Taubheit am zweiten postoperativen Tag: ant. OS } \\
(0 / 1 / 2), \mathrm{n}\end{array}$ & $7 / 8 / 1$ & $7 / 8 / 3$ & 0,64 \\
\hline $\begin{array}{l}\text { Taubheit am zweiten postoperativen Tag: } N \text {. } \\
\text { obturatorius }(0 / 1 / 2), \mathrm{n}\end{array}$ & $16 / 0 / 0$ & $16 / 2 / 0$ & 0,17 \\
\hline
\end{tabular}




\subsection{Kraftgrade nach Janda und Mobilisierung}

Vor der dem Eingriff zeigte sich bei der IP-Gruppe bei 12 Patienten ein Kraftgrad nach Janda im zu operierenden Bein von V, bei einem Patienten von II und bei drei Patienten von I. In der OOP-Gruppe waren es 14 mit einem Kraftgrad V, ein Patient mit einem Kraftgrad IV, zwei Patienten mit einem Kraftgrad III und ein Patient mit einem Kraftgrad II. Nach stattgehabter Operation zeigten 12 Patienten beider Gruppen einen Kraftgrad I auf. Weiterhin zeigte in der IP-Gruppe ein Patient einen Kraftgrad II, zwei Patienten einen Kraftgrad III und ein Patient einen Kraftgrad IV auf. Für die OOP-Gruppe zeigten sich postoperativ drei Patienten mit dem Kraftgrad II und je ein Patient mit den Kraftgraden III, IV und V. Am ersten postoperativen Tag hatten bereits 14 Patienten der OOP-Gruppe und 13 Patienten der IP-Gruppe einen Kraftgrad IV wiedererlangt. In der IP-Gruppe zeigten zudem zwei Patienten einen Kraftgrad II und ein Patient einen Kraftgrad III. In der OOP-Gruppe waren es zudem je zwei Patienten mit einem Kraftgrad II und III. Am zweiten postoperativen Tag hatten bereits 17 Patienten der OOP-Gruppe und 14 Patienten der IP-Gruppe einen Kraftgrad nach Janda von IV wiedererlangt, je ein Patient beider Gruppen einen Kraftgrad III sowie ein Patient der IP-Gruppe einen Kraftgrad II.

Präoperativ erreichten 11 Patienten beider Gruppen einen Mobilisationsgrad III, je ein Patient einen Mobilisationsgrad II und vier Patienten der IP-Gruppe sowie sechs Patienten der OOP-Gruppe einen Mobilisationsgrad I. Postoperativ waren beide Patientengruppen lediglich bis Grad I mobilisierbar. Am ersten postoperativen Tag konnten bereits 13 Patienten der IP-Gruppe und neun Patienten der OOP-Gruppe bis zu einem Grad II mobilisiert werden, drei Patienten der IP-Gruppe und weiter neun Patienten der OOP-Gruppe zu einem Grad I. Ähnliches war am Tag zwei nach der Operation zu beobachten. Hier waren es 13 Patienten der IP-Gruppe und 15 der OOP-Gruppe, die bis zu einem Grad II mobilisiert werden konnten. Drei Patienten der OOP-Gruppe und zwei Patienten der IP-Gruppe wurden bis Grad I mobilisiert. 
Tabelle 9: Kraftgrade nach Janda und Mobilisierungsgrade

\begin{tabular}{cccc}
\hline & $\begin{array}{c}\mathrm{IP} \\
\mathrm{n}=16\end{array}$ & $\begin{array}{c}\text { OOP } \\
\mathrm{n}=18\end{array}$ & $\mathrm{p}$ \\
\hline Janda präoperativ $(0 / 1 / 2 / 3 / 4 / 5), \mathrm{n}$ & $0 / 3 / 1 / 0 / 0 / 12$ & $0 / 0 / 1 / 2 / 1 / 14$ & 0,19 \\
\hline Mobilisation präoperativ $(0 / 1 / 2 / 3), \mathrm{n}$ & $0 / 4 / 1 / 11$ & $0 / 6 / 1 / 11$ & 0,87 \\
\hline Janda postoperativ $(0 / 1 / 2 / 3 / 4 / 5), \mathrm{n}$ & $0 / 12 / 1 / 2 / 1 / 0$ & $0 / 12 / 3 / 1 / 1 / 1$ & 0,69 \\
\hline $\begin{array}{c}\text { Mobilisation postoperativ }(0 / 1 / 2 / 3), \mathrm{n} \\
\text { Janda am ersten postoperativen Tag }\end{array}$ & $0 / 16 / 0 / 0$ & $0 / 18 / 0 / 0$ & \\
\hline$(0 / 1 / 2 / 3 / 4 / 5), \mathrm{n}$ & $0 / 0 / 2 / 1 / 13 / 0$ & $0 / 0 / 2 / 2 / 14 / 0$ & 0,88 \\
\hline $\begin{array}{c}\text { Mobilisation am ersten postoperativen Tag } \\
(0 / 1 / 2 / 3), \mathrm{n}\end{array}$ & $0 / 3 / 13 / 0$ & $0 / 9 / 9 / 0$ & 0,06 \\
\hline $\begin{array}{c}\text { Janda am zweiten postoperativen Tag } \\
(0 / 1 / 2 / 3 / 4 / 5), \mathrm{n}\end{array}$ & $0 / 0 / 1 / 1 / 14 / 0$ & $0 / 0 / 0 / 1 / 17 / 0$ & 0,56 \\
\hline $\begin{array}{c}\text { Mobilisation am zweiten postoperativen Tag } \\
(0 / 1 / 2 / 3), \mathrm{n}\end{array}$ & $0 / 2 / 13 / 1$ & $0 / 3 / 15 / 0$ & 0,54 \\
\hline
\end{tabular}

\subsection{Katheteranlage}

Die Anzahl der Versuche, die zur Platzierung des Katheters nötig waren, unterschied sich in beiden Gruppen kaum (IP-Gruppe 1,1 $( \pm 0,3)$ und OOP-Gruppe 1,2 $( \pm 0,6)$.

Auch die Tiefe des Nervs unter dem Hautniveau bzw. die Eindringtiefe der Nadel war in beiden Gruppen vergleichbar $((5,1 \mathrm{~cm}( \pm 1,5)$ in der IP-Gruppe und 4,8cm $( \pm 1,2$ in der OOP-Gruppe)). Die Einlagetiefe des Katheters war in der OOP-Gruppe mit 9,3cm $( \pm 1,7)$ um $0,7 \mathrm{~cm}$ tiefer als in der IP-Gruppe mit 8,6cm $( \pm 2,8 ; \mathrm{p}=0,33)$. Die Entfernung des Katheters erfolgte in der IP-Gruppe durchschnittlich nach 3,9 Tagen $( \pm 1,0)$ und nach 3,3 Tagen $( \pm 1,4)$ in der OOP-Gruppe.

Tabelle 10: Katheteranlage

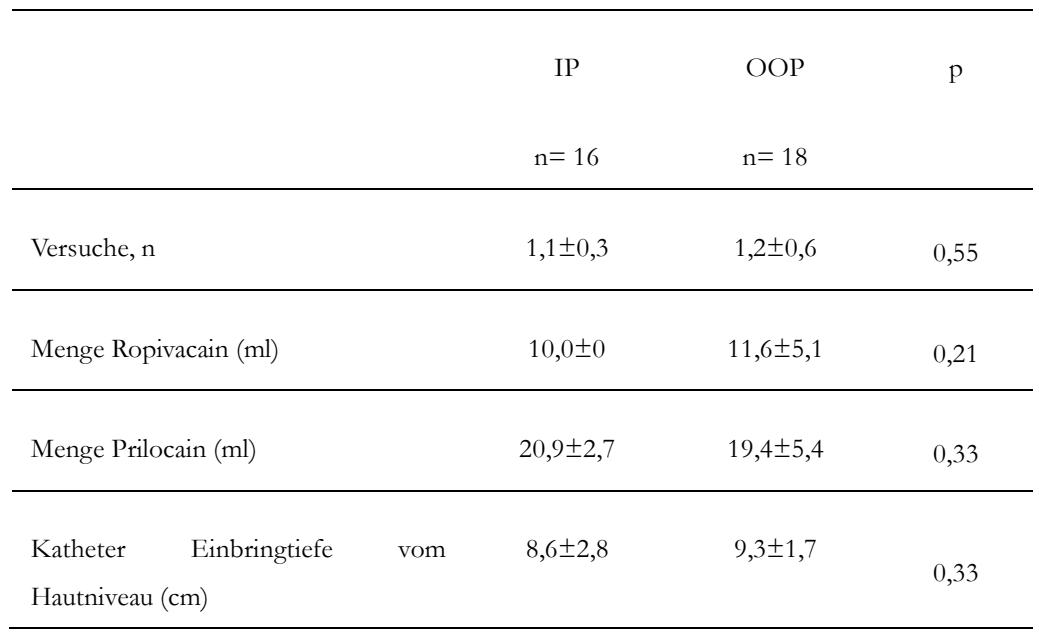




\begin{tabular}{lccc}
\hline Nadeltiefe $(\mathrm{cm})$ & $5,1 \pm 1,5$ & $4,8 \pm 1,2$ & 0,62 \\
\hline Katheter entfernt (Tag) & $3,9 \pm 1,0$ & $3,3 \pm 1,4$ & 0,26 \\
\hline
\end{tabular}

\subsection{Katheterverlauf im Röntgenbild}

Die Auswertung des Nervenkatheterverlaufs konnte bei 13 IP-Patienten und bei 10 Patienten der OOP-Gruppe anhand postoperativer Röntgenaufnahmen am zweiten bis zum fünften postoperativen Tag erfolgen. Im Vergleich beider Gruppen ergaben sich hinsichtlich der Lokalisation der Nervenkatheterspitze innerhalb der Regionen 0, 1a, 1b, 2 und 3 auf den Röntgenaufnahmen keine Unterschiede $(p=0.20)$. Innerhalb der Zielregion 0 lag die Nervenkatheterspitze bei 38.5\% der IP-Patienten und bei 50.0\% der OOP-Patienten. Die außerhalb der Zielregion 0 liegenden Nervenkatheterspitzen waren in beiden Gruppen am häufigsten in Region 3 (distal-medial zur Zielregion) zu sehen (IP: 46.2\%, OOP: 30.0\%). In proximal lokalisierten Regionen waren nur zwei Katheterspitzen aus der OOP-Gruppe (20.0\%, Region 1a) lokalisiert.

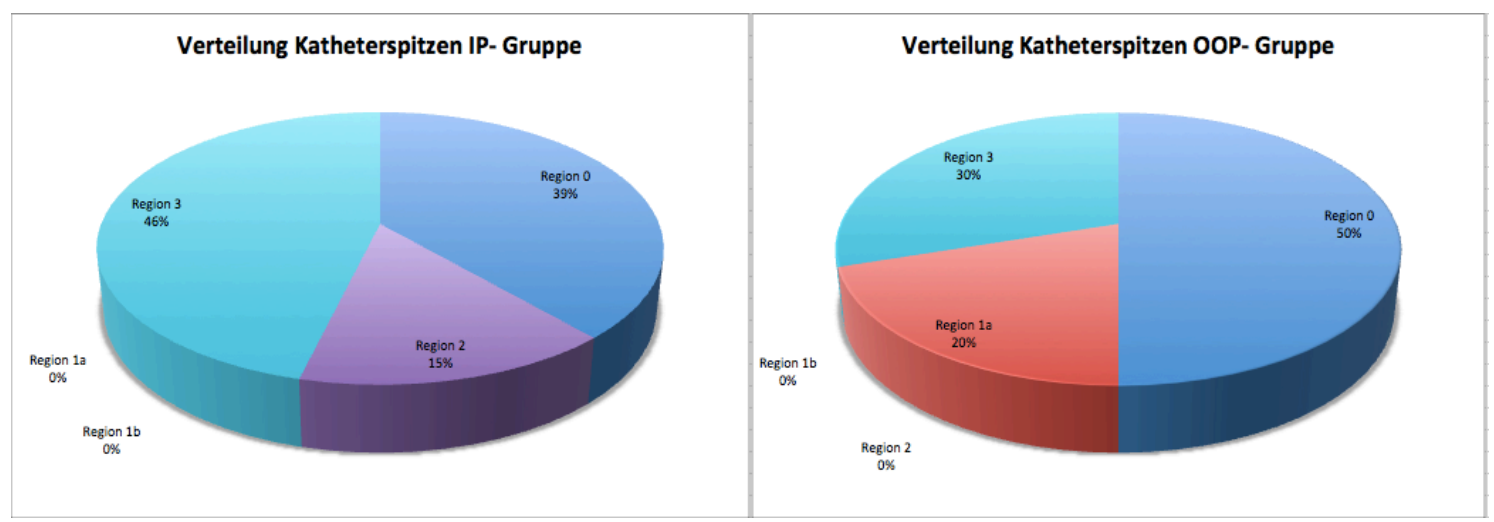

Abbildung 10: Verteilung der Katheterspitzen in der IP- und in der OOP-Gruppe

Die mittlere Spannbreite zwischen der Katheterspitze und der Zielregion war $0.00 \mathrm{~cm} \mathrm{[-}$ 3.80 - 3.84] in der OOP-Gruppe verglichen mit einer deutlichen Abweichung nach distal $1.19 \mathrm{~cm}$ [-12.27 - 0.00] $(\mathrm{p}=0.045)$ in der IP-Gruppe. Die Katheter in der IP-Gruppe waren häufiger nach distal umgeschlagen (IP: 61.5\%, OOP: 10.0\%; $\mathrm{p}=0.01$ ). 
Tabelle 11: Katheterverlauf im Röntgenbild

\begin{tabular}{|c|c|c|c|}
\hline & IP & OOP & $\mathrm{p}$ \\
\hline & $\mathrm{n}=13$ & $\mathrm{n}=10$ & \\
\hline Röntgenbild am 2.POD & $7(53.9 \%)$ & $6(60,0 \%)$ & \\
\hline 3. POD & $4(30.8 \%)$ & $3(30,0 \%)$ & \\
\hline 4. POD & $1(7,7 \%)$ & $1(10,0 \%)$ & \\
\hline 5. POD & $1(7,7 \%)$ & $0(0,0 \%)$ & 0,84 \\
\hline Katheterspitzen in der Zielregion $0, \mathrm{n}$ & $5(38,5 \%)$ & $5(50,0 \%)$ & \\
\hline Region 1a, $\mathrm{n}$ & $0(0,0 \%)$ & $2(20,0 \%)$ & \\
\hline Region $1 \mathrm{~b}, \mathrm{n}$ & $0(0,0 \%)$ & $0(0,0 \%)$ & \\
\hline Region 2, n & $2(15,4 \%)$ & $0(0,0 \%)$ & \\
\hline Region 3, n & $6(46,2 \%)$ & $3(30,0 \%)$ & 0,20 \\
\hline Distanz zur Zielregion, cm & $\begin{array}{c}-1.19[-12.27- \\
0.00]\end{array}$ & $\begin{array}{c}0.0[-3.80- \\
3.84]\end{array}$ & 0,045 \\
\hline Katheter nach distal umgeschlagen, $\mathrm{n}$ & $8(61,5 \%)$ & $1(10.0 \%)$ & 0,01 \\
\hline
\end{tabular}




\section{Diskussion}

In der vorliegenden Studie wurde der Verlauf von röntgendichten Nervus-femoralisKathetern auf der Basis von postoperativen Röntgenbildern des Hüftgelenks evaluiert. Die Katheter wurden mit der In-plane- beziehungsweise mit der Out-of-plane-Methode angelegt. Es zeigte sich, dass die Katheter, die mittels der Out-of-plane-Methode angelegt wurden, mit einer deutlich erhöhten Wahrscheinlichkeit in dem vordefinierten Zielgebiet zu liegen kamen. Die mittels der In-plane-Methode gelegten Katheter zeigten hingegen ein deutlich erhöhtes Risiko, nach distal umzuschlagen. Überraschenderweise zeigte sich, trotz der sehr unterschiedlichen Verläufe der Katheter, kein signifikanter Effekt auf das postoperative Schmerzempfinden innerhalb der beiden Patientengruppen.

\subsection{Bewertung der eigenen Ergebnisse}

Der Grad des postoperativen Schmerzniveaus nach Hüftoperationen beeinflusst maßgeblich die Länge des Krankenhausaufenthaltes und das funktionelle Ergebnis der Operation (Morrison et al. 2003). Die Bedeutung einer adäquaten postoperativen Schmerztherapie wird neben der geringeren Krankenhausverweildauer auch durch ein niedrigeres Morbiditäts- und Mortalitätsrisiko verdeutlicht (Carr und Goudas 1999; Kehlet und Wilmore 2002). Die kontinuierliche Blockade des Nervus femoralis ist eine zuverlässige Form der postoperativen Analgesie nach Hüftoperationen und weist eine geringe Komplikationsrate auf (Capdevila et al. 2005; Richman et al. 2006; Chaudet et al. 2016). Die Katheter können sowohl mittels der In-plane- als auch mittels der Out-of-plane- Methode angelegt werden. Die korrekte Lage der Katheterspitze - nahe des zu betäubenden Nervs - ist dabei von besonderem Interesse, um eine sichere postoperative Analgesie zu gewährleisten. $\mathrm{Zu}$ den häufigsten Problemen zählen die korrekte Anlage sowie die Migration und die Dislokation der Katheterspitze (Elsharkawy et al. 2016). Bislang existieren keinerlei Studien zu der Frage, ob die verschiedenen ultraschallgestützten Anlagetechniken einen Einfluss auf den Verlauf des Katheters, die Lage der Katheterspitze und ein daraus resultierendes verändertes Schmerzempfinden der Patienten haben. In einer Studie von Capdevila et al. wurde die Position der Katheterspitze für 3-in-1-Katheter untersucht. Hierzu wurde über die Katheter ein Röntgenkontrastmittel injiziert und anschließend eine Beckenübersichtsaufnahme angefertigt. Als Anlagetechnik wurde in dieser Studie ausschließlich die Out-ofplane-Methode angewendet. Im Unterschied zu der hier vorliegenden Studie wurden die 
Katheter sehr viel weiter (bis 17,5 cm) über die Nadelspitze hinausgeschoben. Übereinstimmend mit den Ergebnissen dieser Studie befanden sich mehr als 90\% der Katheterspitzen, die in dieser Technik angelegt wurden, proximal des Hüftgelenks, wohingegen ein abweichender Katheterverlauf nur selten auftrat (Capdevila et al. 2002). Die in der vorliegenden Studie angelegten In-plane-Katheter hingegen rotierten zu 60\% entgegen der eigentlich intendierten Zielregion proximal des Hüftkopfes und kamen in der Folge distal zu dieser Region zu liegen. Diese Ergebnisse stützen die These, dass Katheter, die unter Verwendung der In-plane-Technik senkrecht zum Nerven eingebracht wurden, nach Verlassen der Nadel kaum zu kontrollieren sind (Ilfeld et al. 2010).

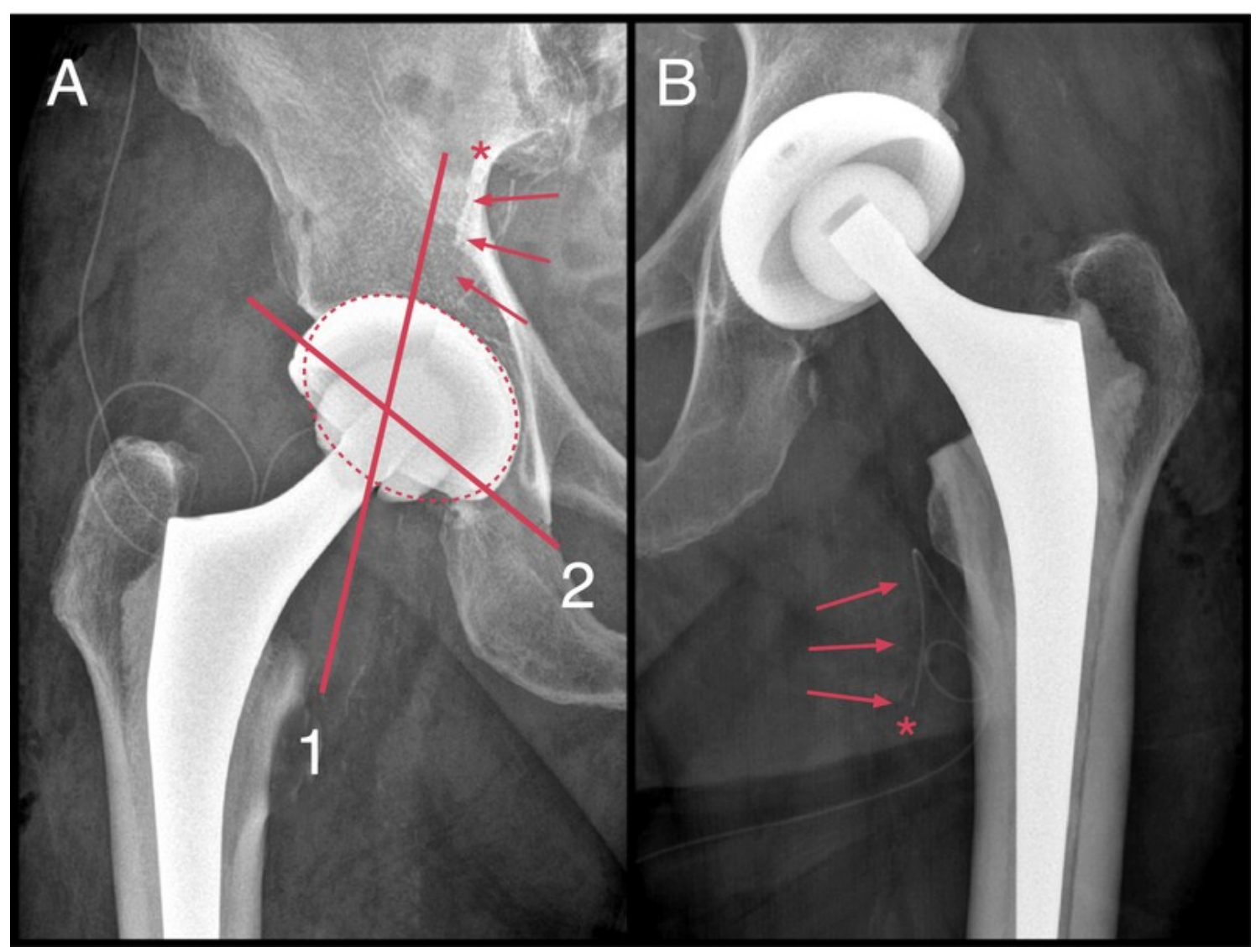

Abbildung 11: (A) OOP-Katheter, (B) IP-Katheter

(A) Die gepunktete Linie markiert die Zielregion, die angrenzenden Regionen werden durch die Linien 1 und 2 geteilt (siehe Material und Methoden). Die Pfeile markieren das Endstück eines OOP-Nervus-femoralis-Katheters. Der Stern markiert das Ende des Nervenkatheters in der Region Nr. 2 proximal der Zielregion.

(B) Die Pfeile markieren das Endstück eines IP-Nervus-femoralis-Katheters, der nach distal umgeschlagen ist. Der Stern markiert das Ende des Nervenkatheters in der Region Nr. 3 deutlich distal der Zielregion.

Da sich der Stamm des Nervus femoralis distal der vordefinierten Zielregion, also distal des Ligamentums inguinale, in seine verschiedenen Äste spaltet, war von einer verminderten 
oder inkompletten Wirkung der neuralen Blockade auszugehen. Überraschenderweise zeigte sich jedoch in beiden Gruppen eine ähnliche Qualität der postoperativen Analgesie und dies, obwohl die Katheterspitzen in der In-plane Gruppe teilweise deutlich von der Zielregion weg rotiert und auch insgesamt signifikant distaler lagen als die Katheter aus der Outof-plane Gruppe (s. Abbildung 11). Diese Erkenntnis deckt sich mit den Ergebnissen zweier weiterer Studien, in denen die Out-of-plane und die In-plane Methode in Bezug auf die postoperative Analgesie für Nervus-femoralis-Katheter verglichen wurden. Auch in diesen Studien konnte kein signifikanter Unterschied zwischen den beiden Gruppen festgestellt werden (Wang et al. 2010; Fredrickson und Danesh-Clough 2013).

Trotz der vermeintlich besseren Platzierung der Katheterspitze zeigte sich am zweiten postoperativen Tag ein leicht erhöhtes Schmerzniveau in der Out-of-plane-Gruppe $(1,9 \pm 1,4)$ im Vergleich zur In-plane-Gruppe $(1,3 \pm 0,6 ; p=0,01)$. Analog zu diesem Ergebnis zeigte die Out-of-plane-Gruppe mit $8,4( \pm 1,8)$ auch eine geringere allgemeine Patientenzufriedenheit als die In-plane-Gruppe mit 9,5( $\pm 0,9 ; \mathrm{p}=0,03)$. Jedoch hatten die Patienten der Out-of-plane-Gruppe bereits präoperativ mit 2,6 $( \pm 1,9)$ ein leicht erhöhtes Schmerzniveau auf der NRS angegeben als die Patienten der In-plane-Gruppe mit 1,6 $\pm 1,2$; $\mathrm{p}=0,05)$. Diese Erklärung wird unterstützt durch die Tatsache, dass in beiden Gruppen ein nahezu identischer Verbrauch an Lokalanästhetika und kein relevanter Unterschied in der Abforderung von PCA-Boli bestand.

Diese Ergebnisse legen nahe, dass die Position der Katheterspitze entlang der mediolateralen bzw. der kranio-kaudalen Achse nicht alleinig ausschlaggebend für den letztendlichen Effekt der postoperativen Analgesie ist. Diese Interpretation wird von zwei Studien geteilt, die die Position von 3-in-1 Kathetern mittels Computertomographie (Ganapathy et al. 1999) und mittels motorischer Antwort bei Nervenstimulation (Pham Dang et al. 2009) untersuchten. In beiden Studien zeigten sich verschiedenste Positionierungen der Katheterspitze, ohne jedoch eine entscheidende Korrelation zwischen der Wirkung und der Lokalisation zu ermöglichen (Ganapathy et al. 1999; Pham Dang et al. 2009).

Mehrere Studien haben diesen Umstand bereits beschrieben und schlussfolgern hieraus, dass eine effektive Blockade des Nervus femoralis vor allem von der Applikation des Lokalanästhetikums in das Kompartiment unter der Fascia iliaca abhängig ist (Dalens et al. 1989; Capdevila et al. 1998; Ganapathy et al. 1999; Capdevila et al. 2002; Fredrickson und Danesh-Clough 2013; Swenson et al. 2015). In diesem Kompartiment liegt der Nervus femoralis direkt unter der Fascia iliaca und verläuft zwischen dem Musculus iliacus und dem Musculus psoas major. Der Nervus cutaneus femoris lateralis verläuft ebenfalls in 
diesem Kompartiment auf der ventralen Seite des Musculus iliacus. Dieser Nerv ist hier von besonderer Bedeutung für das postoperative Schmerzempfinden der Patienten, da sein Innervationsgebiet üblicherweise das des Hautschnittes bei Schenkelhalsoperationen ist. Wie auch bei der herkömmlichen Punktion für einen Fascia iliaca-Block, wurde auch in dieser ultraschallgeführten Studie der Injektionsort durch den „Doppelklick“ unter der Fascia iliaca charakterisiert. Dieser Doppelklick entsteht durch das Durchstechen des Ligamentums inguinale bzw. der Fascia lata und der Fascia iliaca. Auf diesem Wege wird das Lokalanästhetikum direkt in das Kompartiment injiziert und so eine adäquate Analgesie in beiden Gruppen erreicht (Wang et al. 2010; Fredrickson und Danesh-Clough 2013).

Auch im Vergleich mit der rein stimulationsgesteuerten 3-in-1-Blockade erzielt der Fascia iliaca-Kompartimentenblock mit dem Doppelklick-Phänomen häufiger eine kombinierte Blockade des Nervus femoralis und des Nervus cutaneus femoris lateralis, da offenbar bei der stimulationsgesteuerten 3-in-1-Blockade die Fascia iliaca nicht immer sicher perforiert wird und sich das Lokalanästhetikum so nicht entlang der Fascia iliaca vom Nervus femoralis bis zum Nervus cutaneus femoris lateralis in dem Kompartiment verbreiten kann (Capdevila et al. 1998). Im Vergleich der IP- und der OOP-Anlagetechnik für Nervenkatheter liefern unsere Ergebnisse Hinweise darauf, dass die kombinierte Blockade des Nervus femoralis und die des Nervus cutaneus femoris lateralis nach OOP-Anlage häufiger erzielt werden kann als nach IP-Anlage. Eine Erklärung hierfür könnte der unterschiedliche Verlauf der Nervenkatheter sein. In der OOP-Gruppe waren 90\% der Nervenkatheterspitzen nach proximal in Richtung beider Nerven gerichtet und begünstigten somit eine Verteilung des injizierten Lokalanästhetikums unter die Fascia iliaca in Richtung des Nervus femoralis und des Nervus cutaneus femoris lateralis. Im Falle der nach distal umgeschlagenen IP-Katheter dürfte die Verteilung des Lokalanästhetikums nach proximal weniger gut möglich sein. Negative Auswirkungen auf den Wirkungsgrad der Nervenblockade bei Nerven, die in einem weniger klar definierten Faszienkompartiment verlaufen, wie beispielsweise dem Nervus ischiadikus bei einem interskalinären Katheter, scheinen in diesem Kontext wahrscheinlich.

Ein Umschlagen der mittels der IP-Methode gelegten Nervenkatheter nach distal könnte möglicherweise dadurch vermieden werden, dass man die Katheter nur wenige Zentimeter über die Punktionsnadel und damit auch über den Zielnerven hinaus vorschiebt. In einer Untersuchung der IP-Anlage von Nervenkathetern an den Nervus ischiadicus wurden Insertionslängen von 0-1 cm mit einer Insertionslänge von 5-6 cm miteinander verglichen. Hinsichtlich der postoperativen Analgesie konnte hier zwar kein Unterschied beider Methoden nachgewiesen werden, allerdings neigten die nur kurzstreckig eingelegten Nerven- 
katheter eher zur Dislokation (Ilfeld et al. 2011); letzteres dürfte in der Femoralregion, in der ein Nervenkatheter im Gegensatz zu der den Nervus ischiadicus umgebenden Muskulatur nahezu ausschließlich durch Haut und subkutanes Gewebe fixiert wird, noch häufiger vorkommen.

In vorausgegangenen Studien wurde die Möglichkeit einer erhöhten Wahrscheinlichkeit neuraler Verletzungen unter Verwendung der OOP-Methode diskutiert. Diese Annahme beruht auf der nur punktuell einsehbaren Spitze der Nadel, wohingegen unter Verwendung der IP-Methode die Nadelspitze sowie die Zielstruktur jederzeit voll einsehbar sind. Eine versehentliche Punktion des Nervs und eine damit einhergehende Schädigung scheinen deshalb unter Verwendung der OOP-Methode wahrscheinlicher. In einer Studie von Ruiz et al. konnte auch tatsächlich eine erhöhte Rate von Nadel-Nerven-Kontakten in der OOPGruppe (64\% der Fälle) im Gegensatz zur IP-Gruppe (9\% der Fälle) festgestellt werden. Diese beobachteten Nadel-Nerven-Kontakte führten jedoch weder in der IP- noch in der OOP-Gruppe zu bleibenden Nervenschädigungen (Ruiz et al. 2014). Ein Nadel-NervKontakt wurde in der hier vorliegenden Studie zwar nicht erfasst, jedoch wurden nach Beendigung des Katheterverfahrens auch hier weder in der IP- noch in der OOP-Gruppe Folgeschäden im Innervationsgebiet des Nervus femoralis beobachtet.

Beide Studiengruppen waren hinsichtlich der Basisparameter wie Alter, Geschlecht, Größe, Gewicht, ASA-Klassifikation, NYHA-Klassifikation, Art des Anästhesieverfahrens und hinsichtlich der Dringlichkeit des operativen Eingriffs miteinander vergleichbar. Die Übereinstimmung dieser Parameter ermöglicht die Vergleichbarkeit der beiden Gruppen hinsichtlich subjektiver Parameter, wie des postoperativen Schmerzempfindens. Um einen Qualitätsunterschied zwischen den beiden hier untersuchten Techniken zur Anlage eines Nervus-femoralis-Katheters darstellen zu können, wurden im Vorfeld mögliche Störfaktoren reduziert. Die Patienten wurden über die beiden unterschiedlichen Techniken aufgeklärt, eine Wahlmöglichkeit zwischen den beiden Verfahren bestand jedoch nicht. Es erfolgte eine Randomisierung der Patienten in die beiden Studiengruppen, um eine Befangenheit einer der beiden Methoden gegenüber zu vermeiden. Die Anlage der Katheter erfolgte in der zentralen Einleitung und wurde durch insgesamt vier Studienärzte durchgeführt. Alle Studienärzte verfügten über ein fundiertes Wissen und ausreichend Erfahrung in der Regionalanästhesie, insbesondere mit den hier verwendeten Techniken, so dass eine Vergleichbarkeit in der Qualität der Katheteranlage gewährleistet war. Die Auswertung der postoperativen Röntgenbilder erfolgte durch den Doktoranden in Zusammenarbeit mit einem radiologischen Oberarzt, um möglichst genaue Messungen zu generieren. 
Die Anlage der Katheter erfolgte in beiden Gruppen problemlos, ohne dass mehrere Versuche dafür notwendig gewesen wären. Die Nadeltiefe gibt die Entfernung vom Hautniveau bis zum Nerven an. In der IP-Gruppe lag der Nerv im Mittel bei 5,1 $11,5 \mathrm{~cm}$ und in der OOP-Gruppe bei 4,8 $\pm 1,2 \mathrm{~cm}$. Der Katheter wurde in der IP-Gruppe durchschnittlich $8,6 \pm 2,8 \mathrm{~cm}$ und in der OOP-Gruppe $9,3 \pm 1,7 \mathrm{~cm}$ tief eingebracht $(\mathrm{p}=0,33)$. Hieraus ergibt sich ein netto Unterschied von $1 \mathrm{~cm}$. Eine geringere Eindringtiefe des Katheters könnte vor allem durch die geringere Fixation im Gewebe und durch Mobilisierungsmaßnahmen zu einer höheren Rate an Dislokationen führen (Ilfeld et al. 2011). Die Katheter wurden in der IP-Gruppe durchschnittlich nach 3,9 $\pm 1,0$ und nach 3,3, $\pm 1,4$ Tagen in der OOP-Gruppe entfernt. Dislokationen wurden dabei nicht beobachtet.

Präoperativ wurde von keinem der Patienten ein Taubheitsgefühl in der zu operierenden Extremität angegeben. In der Auswertung der Daten zeigte sich, dass direkt postoperativ, bis auf je einen Patienten der IP- und einen Patienten der OOP-Gruppe, alle Patienten eine Parästhesie des ventralen und des lateralen Oberschenkels verspürten. Am zweiten postoperativen Tag gaben immerhin noch 11 Patienten der OOP-Gruppe und 9 Patienten der IP-Gruppe Taubheitsgefühle im Bereich des lateralen und des ventralen Oberschenkels an. Eine Taubheit im Versorgungsgebiet des Nervus obturatorius war bei der in dieser Studie angewendeten Technik des reinen Nervus-femoralis-Katheters nicht zu erwarten. Dass bei der hier vorliegenden Studie dennoch vor allem postoperativ bei 11 Patienten der OOPGruppe und bei immerhin 8 Patienten der IP-Gruppe ein Effekt auf den Nerven zu beobachten war, ist in diesen Fällen wohl vor allem auf dem immer noch erhaltenen Effekt der Spinalanästhesie begründet. Am zweiten postoperativen Tag konnte eine Taubheit im Innervationsgebiet des Nervus obturatorius bei keinem Patienten der IP-Gruppe und lediglich bei zwei Patienten der OOP-Gruppe nachgewiesen werden. Da die Katheter der OOPGruppe auch insgesamt weiter nach proximal reichten, kam es in diesen beiden Fällen wohl zu einer teilweisen Mitwirkung des Katheters auf den Plexus lumbalis.

Neben den Parästhesien wurden auch die NRS-Werte präoperativ bis zum zweiten postoperativen Tag erhoben, um so weitere Rückschlüsse auf die Qualität der Nervenblockade zu erhalten. Die subjektive Bewertung des Schmerzes durch den Patienten ist hierbei nicht unkritisch zu sehen und wurde deshalb im Zusammenspiel mit den abgeforderten PCABoli und dem angegebenen Taubheitsgefühl in der operierten Extremität beurteilt. So zeigen die Patienten intraindividuell starke Schwankungen im subjektiven Schmerzempfinden. Auch werden die Studienpatienten häufiger visitiert und nach ihrem Befinden befragt. Betagtere Patienten haben zudem häufig Probleme bei der Anwendung der Schmerzskala und können sich leicht unter Druck gesetzt fühlen, vermeintlich richtige Antworten geben zu 
müssen. Die Vergleichbarkeit und Anwendbarkeit der NRS für klinische Studien wird deshalb kritisch betrachtet (Hartrick et al. 2003). Beide Gruppen erhielten ein standardisiertes orales Analgetikaregime, bestehend aus drei mal $600 \mathrm{mg}$ Targin, sowie zwei mal 10/5 mg Targin am Tag. Wie bereits erwähnt zeigte sich in der OOP-Gruppe bereits präoperativ ein erhöhtes Schmerzniveau in Ruhe mit 2,6 ( $\pm 1,9)$ im Gegensatz zur IP-Gruppe mit 1,6 $( \pm 1,2 ; p=0,05)$. Diese Diskrepanz beider Gruppen setzte sich für die Dauer der Untersuchung fort und blieb auf einem konstanten Niveau. Die NRS-Werte in Bewegung verhielten sich dazu proportional $(2,9 \pm 1,8$ in der OOP-Gruppe und 2,0 $\pm 1,2$ in der IP-Gruppe am zweiten postoperativen Tag p=0,15). Diese Ergebnisse decken sich mit den Studien, die bereits die IP- und die OOP-Technik bezüglich ihrer postoperativen Analgesie verglichen haben (Wang et al. 2010; Fredrickson und Danesh-Clough 2013).

Einen weiteren Indikator, um die Qualität der Nervenblockade zu quantifizieren, bieten in dieser Studie die Laufrate sowie die abgeforderten Boli aus den PCA-Pumpen. Die basale Laufrate variierte zwischen den Gruppen kaum und lag am zweiten postoperativen Tag nahezu identisch in der IP-Gruppe bei 4,0 $( \pm 3,5)$ und in der OOP-Gruppe bei 3,7 ( $\pm 3,2$; $\mathrm{p}=0,81)$. Auch die Anzahl abgeforderter Ropivacain-Boli variierte zunächst nicht $(5,5 \pm 4,2$ in der IP-Gruppe und 5,3 \pm 7,0 in der OOP-Gruppe am ersten postoperativen Tag $\mathrm{p}=0,89$ ). Am zweiten postoperativen Tag zeigte sich jedoch ein Anstieg in der Menge der abgeforderten Boli in der IP-Gruppe mit 3,4 $\pm 4,5$ im Gegensatz zu OOP-Gruppe mit 2,2 $\pm 3,6$ abgeforderten Boli. Interessanterweise geschah dies trotz oder gerade wegen des erhöhten Schmerzniveaus in der OOP-Gruppe.

Ein weiterer Parameter, der in dieser Studie Berücksichtigung fand, ist die allgemeine Patientenzufriedenheit. Sie wurde ebenfalls mit einer NRS (1-10) erhoben. Sie bietet aber, wie auch die Schmerzskala, aufgrund der vielen Einfluss- und Störfaktoren höchstens einen indirekten Blick auf die Qualität der Analgesie (Avis et al. 1995). Die Ausgangswerte der mittleren Patientenzufriedenheit lagen in der IP-Gruppe bei 8,5 $\pm 1,2$ und 8,1 $\pm 2,3$ in der OOP-Gruppe $(\mathrm{p}=0,5)$. Bereits am ersten postoperativen Tag zeigte eine Verschlechterung in der OOP-Gruppe auf 7,6 2,6, wohingegen die Zufriedenheit in der IP-Gruppe sogar zunahm $(9,3 \pm 1,0 ; \mathrm{p}=0,02)$. Am zweiten postoperativen Tag erholte sich auch die Patientenzufriedenheit in der OOP-Gruppe und stieg mit 8,4 $\pm 1,8$ sogar über das Ausgangs-niveau, blieb aber dennoch hinter dem der IP-Gruppe mit 9,5 $\pm 1,8$ zurück $\mathrm{p}=0,03$. Auch in der von Fredrickson und Danesh-Clough durchgeführten vergleichenden Studie zu In- und Out-ofplane-Nervus-femoralis-Katheteranlagen zeigte sich ein ähnlicher Unterschied in der Patientenzufriedenheit. Hier lag die Patientenzufriedenheit allerdings in der OOP-Gruppe, mit 
durchschnittlich 10 Punkten auf der NRS, höher als in der IP-Gruppe mit durchschnittlich 9 Punkten (Fredrickson und Danesh-Clough 2013).

Durch die handelsüblichen Lokalanästhetika werden nicht nur sensorische (afferente), sondern auch motorische (efferente) Neuronen beeinträchtigt (Ilfeld und Yaksh 2009). Eine Muskelschwäche des Musculus quadriceps femoris ist die Folge (Salinas 2004). Durch diese Muskelschwäche ist eine erhöhte Sturzneigung vor allem bei älteren Patienten zu beobachten (Stevens et al. 2003). Bei den im Rahmen dieser Studie untersuchten Patienten ereigneten sich in der Mobilisationsphase keinerlei Stürze. Aufgrund der durch den Nervusfemoralis-Katheter verursachten muskulären Schwäche wurde neben dem Kraftgrad nach Janda auch der Mobilisationsgrad bestimmt. Bezogen auf den Kraftgrad nach Janda ergaben sich innerhalb der beiden Gruppen kaum Unterschiede. So erlangten in der IP-Gruppe 14 von 16 Patienten und in der OOP-Gruppe 17 von 18 Patienten am zweiten postoperativen Tag bereits einen Kraftgrad 4 nach Janda (Bewegung gegen mäßigen Widerstand). Für die Mobilisationsgrade stellt sich hier ein ähnliches Bild dar. Am zweiten postoperativen Tag konnten 13/16 Patienten der IP-Gruppe und 15/18 der OOP-Gruppe bereits bis zum Mobilisationsgrad 2 (eingeschränktes Gehen) mobilisiert werden. Hieraus ergab sich kein Anhalt für einen höheren paretischen Effekt eines der beiden Verfahren auf die Muskeln im Innervationsgebiet des Nervus femoralis.

Bei der Analyse der Röntgenbilder zeigten sich deutliche Unterschiede im Verlauf der Katheter im Gewebe. Als besonders auffällig ist hier zu erwähnen, dass die Katheter der IPGruppe im Mittel 1,19 cm distal der Zielregion zu liegen kamen, wohingegen die Katheter der OOP-Gruppe im Mittel die Zielregion erreichten $\mathrm{p}=0.045$. Dieser Umstand lässt sich vor allem durch die hohe Rate an nach distal umgeschlagenen Kathetern in der IP-Gruppe erklären. So befanden sich in dieser Gruppe 8 (61,5\%) Katheterspitzen in einer mindestens $90^{\circ}$ Abweichung von der Linie 1. In der OOP-Gruppe konnte dies nur in einem einzigen Fall beobachtet werden $p=0,01$. Insgesamt zeigte sich hier, dass diese 8 von der eigentlich intendierten Richtung abgewichenen Katheter allesamt in den Regionen 2 und 3 zu liegen kamen und somit deutlich distal des Zielgebietes.

In dieser Studie konnte gezeigt werden, dass es bei der ultraschallgestützten Anlage von Nervus-femoralis-Kathetern in der IP-Technik - im Gegensatz zur OOP-Technik - deutlich häufiger zu einem Umschlagen der Katheterspitze nach distal kommt. Dadurch kommt es auch insgesamt zu einer Abweichung der Katheterspitzen nach distal von dem hier vordefinierten Zielgebiet in der IP-Gruppe. In Bezug auf die sekundären Endpunkte dieser Studie ließen sich dennoch keine relevanten Unterschiede zwischen den beiden Gruppen fest- 
stellen. Eine mögliche Erklärung für diesen Umstand scheint die Lage der Katheterspitze innerhalb des Fascia iliaca-Kompartiments und der damit verbundenen Ausbreitung des Lokalanästhetikums nach kranial. Da so der radiologische Befund in keinen Zusammenhang mit der postoperativen Analgesie gebracht werden kann, konnte für die klinische Praxis kein klarer Vorteil für die ultraschallgestützte Anlage von IP- oder OOP-Kathetern nachgewiesen werden.

\subsection{Methodenkritik und Fehlermöglichkeiten}

Die Patientengruppen waren bezüglich der allgemeinen Patientendaten wie Alter, Geschlecht, Körpergröße und Körpergewicht, NYHA- und ASA-Klassifikation sowie mit der Art der Operation und des gewählten Anästhesieverfahrens vergleichbar. Die Aussagekraft der Studie wird jedoch durch die geringe Zahl an Studienteilnehmern limitiert.

Nervengewebe als solches ist im Röntgenbild nicht darstellbar. Deshalb wurde das Zielgebiet, in dem der Nerv aller Wahrscheinlichkeit nach verläuft, durch die Loge zwischen dem Musculus iliacus und dem Musculus psoas major im Bereich des Hüftkopfes definiert. Anomalien im Nervenverlauf oder eine exakte Beziehung zwischen Nerv und Katheter konnten so jedoch nicht ermittelt werden.

Für diese Studie wurden SonoLong Katheter der Firma Pajunk verwendet. Diese sind der aufgrund der im Inneren des Katheters verarbeiteten Edelstahlspirale im Röntgenbild darstellbar. Für die IP-Gruppe wurden Tuohy-Nadeln, für die OOP-Gruppe Nadeln mit einem Facettenschliff verwendet. Die Verwendung anderer Katheter und Nadeltypen wie z. B. einer Sprottennadel könnten zu anderen Ergebnissen führen.

Die erhobenen Daten bezüglich des Schmerzempfindens, der allgemeinen Patientenzufriedenheit und des Taubheitsempfindens sind stark subjektiv geprägt. Ein Rückschluss auf die tatsächliche Effektivität der Nervenblockade muss deshalb unter Betrachtung aller erhobenen Parameter inklusive der Laufrate und der abgeforderten Boli aus den PCA-Pumpen erfolgen. 


\section{$5 \quad$ Zusammenfassung}

Die vorliegende Studie vergleicht zwei ultraschallgestützte Verfahren zur Anlage eines Nervus-femoralis-Katheters. Eine perioperative Anlage eines Nervus-femoralis-Katheters ist ein weithin etabliertes Verfahren zur postoperativen Analgesie nach Operationen des Hüftgelenks und des proximalen Femurs. Durch die Anlage von röntgendichten Kathetern jeweils in der In-plane-Technik bzw. in der Out-of-plane-Technik, konnte der Verlauf der Katheter im Gewebe im postoperativen Röntgenbild sichtbar gemacht werden. Dazu wurden 34 Patienten randomisiert einer der beiden Gruppen zugeteilt und im Rahmen einer Hüftoperation mit einem Nervus-femoralis-Katheter versorgt. Die beiden Patientengruppen waren bezüglich der allgemeinen Patientendaten wie Alter und Geschlecht etc. miteinander vergleichbar. Die Anlage des Nervus-femoralis-Katheters erfolgte perioperativ in einer zentralen Einleitung durch einen der vier Studienärzte. Postoperativ erhielten die Patienten eine PCA-Pumpe. Die Pumpen ermöglichten den Patienten, neben einer basalen Laufrate auch sich selbst Lokalanästhetika-Boli zu verabreichen. Die Patienten wurden präoperativ, postoperativ und an den nachfolgenden zwei Tagen visitiert. Es wurden täglich Daten bezüglich des Schmerzempfindens, der allgemeinen Zufriedenheit, Taubheitsempfinden an verschiedenen Stellen des Oberschenkels, Kraft- und Mobilisierungsrades, basaler Laufrate und abgeforderter Boli aus den PCA-Pumpen erhoben. Bei der Anlage der Katheter wurde die Nerventiefe, der Vorschub des Katheters über die Nadelspitze und die verwendeten Lokalanästhetika dokumentiert. Zumeist am zweiten postoperativen Tag erfolgte das Entfernen der Wunddrainagen durch die Kollegen der Unfallchirurgie und im Anschluss das postoperative Röntgenbild.

Um den Verlauf des Katheters im Röntgenbild in Bezug zum Verlauf des Nervus femoralis in Beziehung setzen zu können, wurde ein Zielgebiet definiert. Dieses Zielgebiet befindet sich über dem Hüftkopf, da dort der Nervus femoralis zwischen dem Musculus iliacus und dem Musculus psoas major, die beide zum Trochanter minor des Femurs ziehen, verläuft. Das Zielgebiet wurde in 5 Regionen eingeteilt, um eine Beschreibung der letztendlichen Lage der Katheterspitze zu ermöglichen. Die Auswertung des postoperativen Röntgenbildes erfolgte zusammen mit einem Oberarzt der Radiologie.

Bei der Auswertung der Röntgenbilder zeigte sich, dass 60\% der Katheter, die mittels der IP-Technik angelegt wurden, nach distal entgegen der eigentlich intendierten Richtung rotiert waren. In der OOP-Gruppe war dies nur bei einem Katheter der Fall. Diese Beobach- 
tung stützt die These, die bereits in vorrausgegangenen Studien formuliert wurde, dass IPKatheter nach Verlassen der Nadelspitze unkontrollierbar im Gewebe vorgeschoben werden, da diese über einen Winkel eingebracht werden müssen. Auch der Abstand zur Zielregion war für die IP-Katheter insgesamt nach distal verschoben. Entgegen den Erwartungen aus diesem Ergebnis zeigten sich für die sekundären Endpunkte keine relevanten Unterschiede in den beiden Patientengruppen. In Bezug auf Taubheitsempfinden, Mobilisierung und Kraftgrad waren beide Gruppen postoperativ miteinander vergleichbar. Ein leicht erhöhtes Schmerzniveau in der OOP-Gruppe bestand bereits präoperativ und blieb für die Dauer der Studie bestehen. Die gute Wirkung der nach distal umgeschlagenen Katheter wurde, wie in vorrausgegangenen Studien bereits postuliert, über die Lage der Katheter innerhalb des Fascia iliaca-Kompartimentes erklärt. Da der radiologische Befund nicht mit der klinischen Praxis korreliert werden konnte, erwachsen aus dieser Studie keine eindeutigen Handlungsempfehlungen für die Wahl zwischen der IP- und der OOP-Technik zur Anlage von Nervus-femoralis-Kathetern. 


\section{$6 \quad$ Anhang}

\subsection{Studienprotokoll}

\section{Präoperativ:}

Gruppe:

Diagnose:

Operation:

ASA:

Narkoseverfahren:

Chron. Schmerzen:

Schmerzmedikati-

on:

Größe:

Gewicht:

\section{Postoperativ:}

\begin{tabular}{|l|l|l|l|l|l|l|l|l|l|l|}
\hline & NRS & $\begin{array}{l}\text { NRS } \\
\text { bei } \\
\text { Be- } \\
\text { weg. }\end{array}$ & $\begin{array}{l}\text { Moto- } \\
\text { rik } \\
\text { nach } \\
\text { Janda }\end{array}$ & $\begin{array}{l}\text { Mobi- } \\
\text { li- } \\
\text { sie- } \\
\text { rung }\end{array}$ & $\begin{array}{l}\text { Taub- } \\
\text { heit } \\
\text { lat. OS }\end{array}$ & $\begin{array}{l}\text { Taub- } \\
\text { heit } \\
\text { med. } \\
\text { OS }\end{array}$ & $\begin{array}{l}\text { Taub- } \\
\text { heit } \\
\text { Obtu- } \\
\text { rat. }\end{array}$ & $\begin{array}{l}\text { Pat. } \\
\text { Zu- } \\
\text { frie- } \\
\text { den- } \\
\text { heit 1- } \\
10\end{array}$ & $\begin{array}{l}\text { Lauf- } \\
\text { rate/ } \\
\text { Ge- } \\
\text { sammt } \\
\text { Pumpe }\end{array}$ & $\begin{array}{l}\text { Boli } \\
\text { Pumpe }\end{array}$ \\
\hline $\begin{array}{l}\text { Prä } \\
\text { OP }\end{array}$ & & & & & & & & & & \\
\hline $\begin{array}{l}\text { OP } \\
\text { Tag }\end{array}$ & & & & & & & & & & \\
\hline $\begin{array}{l}\text { Post } \\
\text { OP 1 }\end{array}$ & & & & & & & & & & \\
\hline $\begin{array}{l}\text { Post } \\
\text { OP 2 }\end{array}$ & & & & & & & & & & \\
\hline
\end{tabular}

Zusatz Schmerzmedikation:

PONV:

Delir:

Sturz:

Infektion Einstichstelle:

Datum Rö:

Lagebeschreibung:

Katheter Anlage:

Anzahl Versuche: kontr. 
Prilo:

Sonst:

\subsection{Patientenaufklärung}

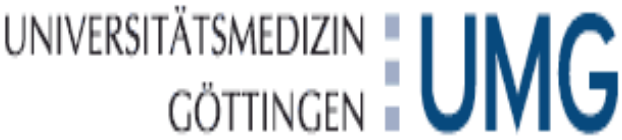

Sehr geehrte Patientin, sehr geehrter Patient,

wir möchten Sie um Ihre freiwillige Teilnahme an der im Folgenden erklärten wissenschaftlichen Studie bitten. Ein Widerruf Ihrer Einverständniserklärung ist jederzeit möglich.

\section{Warum wird diese Studie durchgeführt?}

Dies ist eine wissenschaftliche Untersuchung von zwei etablierten Verfahren zur Anlage eines Schmerzkatheters in die Leiste um Ihnen nach Ihrem Schenkelhalsbruch die Schmerzen zu nehmen. Wir wollen durch diese Studie herausfinden, ob eine der beiden Techniken, die wir mit einem Ultraschallgerät durchführen, vorteilhafter ist.

Für die Versorgung Ihres Schenkelhalsbruches wird Ihnen vor der Operation ein großer Nerv, der hauptsächlich für die Schmerzen im Hüftgelenk verantwortlich ist betäubt. Dies ist für Sie sowohl bereits vor Ihrer Operation wichtig, damit Sie z.B. bei der Umlagerung auf den OPTisch keine Schmerzen haben. Aber auch nach der Operation kommt Ihnen diese Schmerzausschaltung zugute. Sie brauchen dann so gut wie keine weiteren Schmerzmitte mehr. Für die eigentliche Operation erhalten Sie eine Vollnarkose.

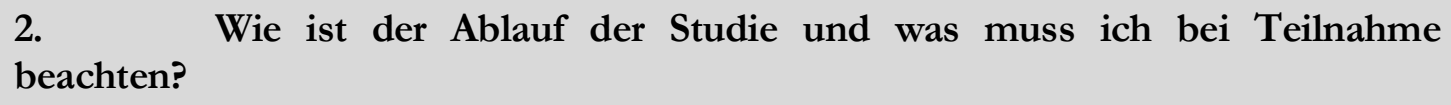

Die Teilnahme an dieser Studie stellt für Sie als Patient keine gesundheitliche Gefahr dar. Während Ihrer geplanten Operation werden Sie von einem/einer Anästhesist/in ständig unter ärztlicher Beobachtung begleitet. Es werden bei Ihnen keine zusätzlichen Maßnahmen zur Durchführung dieser Studie durchgeführt. Die Informationen, die wir für diese Studie benötigen, bekommen wir alleine durch Beobachtung Ihrer Narkose. Dabei dokumentieren wir unter anderen Ihre Werte für Blutverlust, Operationsdauer, sowie der Entlassungszeitpunkt nach der Operation. Im Anschluss an den Eingriff erhalten Sie von uns einen Fragebogen, wo Sie uns bitte einige Fragen zu der Anästhesie beantworten. Nach 
2 Tagen werden wir Ihnen noch einmal einen solchen standardisierten Fragebogen vorlegen.

3.

Welchen persönlichen Nutzen habe ich von der Teilnahme an der Studie?

Sie persönlich werden keinen zusätzlichen Nutzen durch die Teilnahme erfahren; allerdings auch keine zusätzliche Belastung oder zusätzliches Risiko. Die Ergebnisse der Studie können aber möglicherweise dazu beitragen, uns zu zeigen, welches Anlageverfahren für die Ausschaltung dieses Nerven am Oberschenkel am besten geeignet ist.

\section{Welche Risiken sind mit der Teilnahme an der Studie verbunden?}

Mit der Teilnahme an dieser Studie sind keine weiteren Risiken verbunden.

\section{Kann meine Teilnahme an der Studie vorzeitig beendet werden?}

Sie können jederzeit, auch ohne Angabe von Gründen, Ihre Teilnahme beenden, ohne dass Ihnen dadurch irgendwelche Nachteile bei Ihrer medizinischen Behandlung entstehen.

Sofern Sie sich dazu entschließen, vorzeitig aus der Studie auszuscheiden, müssen Sie es nur dem/der behandelnden Arzt/Ärztin mitteilen.

\section{Was geschieht mit meinen Daten?}

Die aufgezeichneten, personenbezogenen Daten werden absolut vertraulich und unter Ausschluss der Öffentlichkeit nur für Zwecke der Studie verwendet. Die Daten werden nicht an dritte Personen ausgehändigt. Außerdem werden die erfassten Daten in einer pseudonymisierten Form (Pseudonymisierung bedeutet Verschlüsselung von Daten ohne Namensnennung, nur mit Nummern codiert. Die Zuordnung der Daten zu einer Person ist nur möglich, wenn hierfür der Schlüssel eingesetzt wird, mit dem die Daten pseudonymisiert wurden. Die personenbezogenen Daten werden unter besonderen Schutzvorkehrungen getrennt von den pseudonymisierten Daten aufbewahrt. Eine Entschlüsselung ist nur durch die verantwortlichen Studienärzte möglich. Dritte erhalten keinen Einblick in die Originalunterlagen) mit einer Codenummer gespeichert, die nicht der Person zuzuordnen ist, so dass diese für dritte Personen nicht erkenntlich sind. Die Datenspeicherungszeit ist auf 10 Jahre festgelegt.

Bei Widerruf der Studienteilnahme können Sie die Löschung ihrer personenbezogenen Daten verlangen. 
7. An wen wende ich mich bei weiteren Fragen?

Bei weiteren Fragen können Sie sich an den Projektleiter diese Studie, Dr. Ingo Bergmann (Tel.: 0176/80260267), wenden.

Universitätsmedizin Göttingen

Robert-Koch-Str. 4037099 Göttingen

Zentrum Anaesthesiologie, Rettungs- und Intensivmedizin

Direktor: Prof. Dr. med. M. Quintel

Projektleiter:

Dr. Dr. med. Dr. med. I. Bergmann (Tel.: 0176/80260267) 
Einverständniserklärung des Patienten

zur Studie:

„Vergleichende Studie zur Inline und Out of Line Technik, bei Anlage eines Nervus- femoralis-Katheters für Patienten mit Schenkelhalsfraktur, in Hinblick auf die Qualität des Anlageverfahrens und das postoperative Schmerzmanagement"

\section{Patientenaufkleber}

\section{Name, Vorname in Druckbuchstaben}

Ich wurde von dem behandelnden Arzt vollständig über Wesen, Bedeutung und Tragweite der klinischen Prüfung mit dem o.g. Titel aufgeklärt. Ich habe die Patienteninformation gelesen und verstanden. Ich hatte die Möglichkeit, Fragen zu stellen, und habe die Antworten verstanden. Der behandelnde Arzt hat mich über die mit der Teilnahme an der Studie verbundenen Risiken und den möglichen Nutzen informiert. Ich hatte ausreichend Zeit, mich zu informieren. Ich weiß, dass ich die Erklärung jederzeit und ohne Angaben von Gründen diese Zustimmung widerrufen kann, ohne dass sich dieser Entschluss nachteilig auf meine spätere Behandlung auswirken wird.

Mir ist bekannt, dass bei dieser klinischen Prüfung personenbezogene Daten, insbesondere medizinische Befunde, über mich erhoben und pseudonymisiert gespeichert und ausgewertet werden. Die Daten werden nicht an Dritte weitergegeben. Bei Widerruf der Studienteilnahme kann ich die Löschung meiner personenbezogenen Daten verlangen.

Ich habe eine Kopie der Patienteninformation und dieser Erklärung erhalten. Ich erkläre mich hiermit bereit, an dieser klinischen Studie teilzunehmen.

Ort und Datum

Unterschrift des Patienten 
Ort und Datum

Unterschrift des aufklärenden Arztes 


\subsection{Anlageprotokoll}

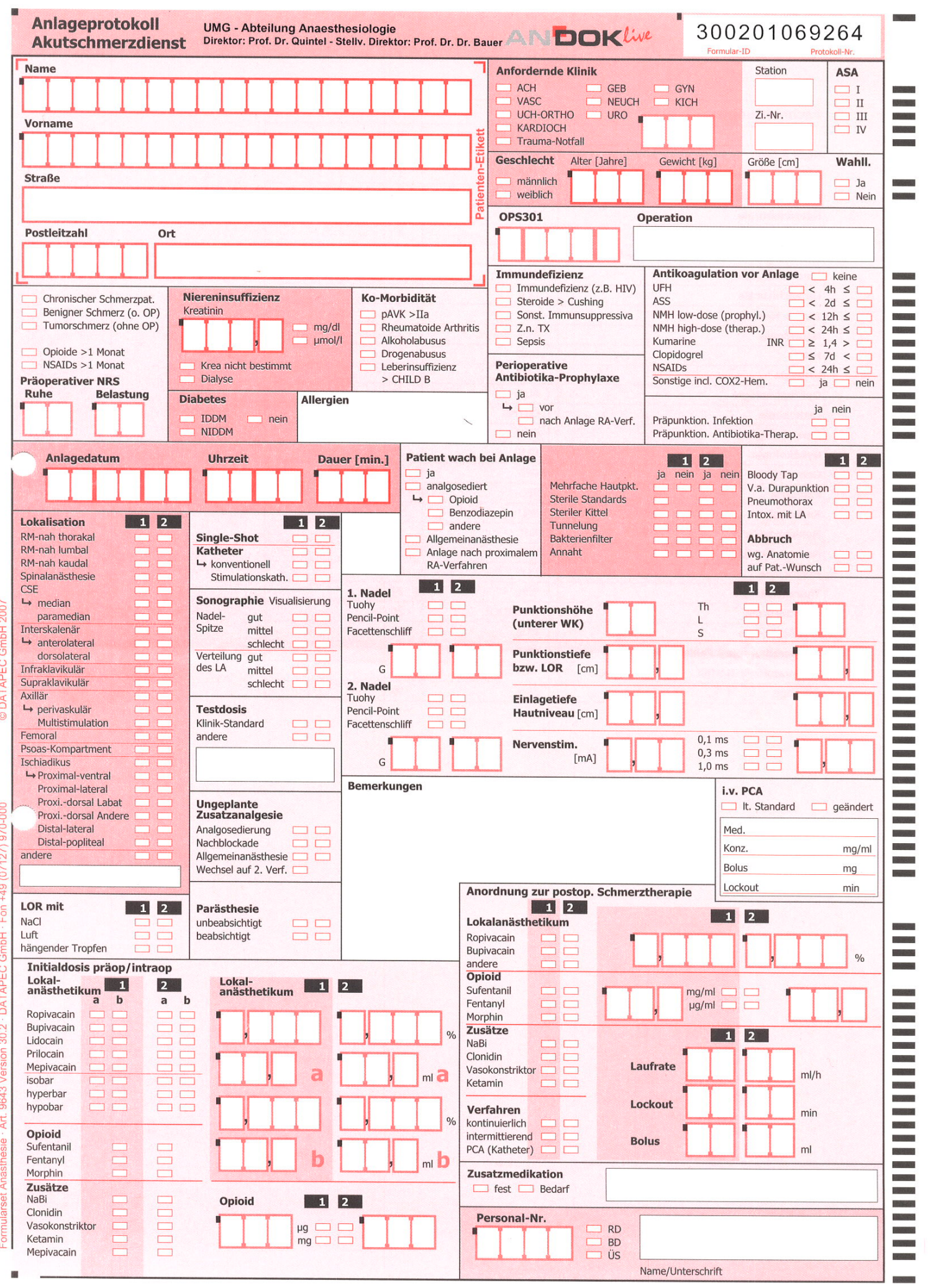




\subsection{Visitenprotokoll}

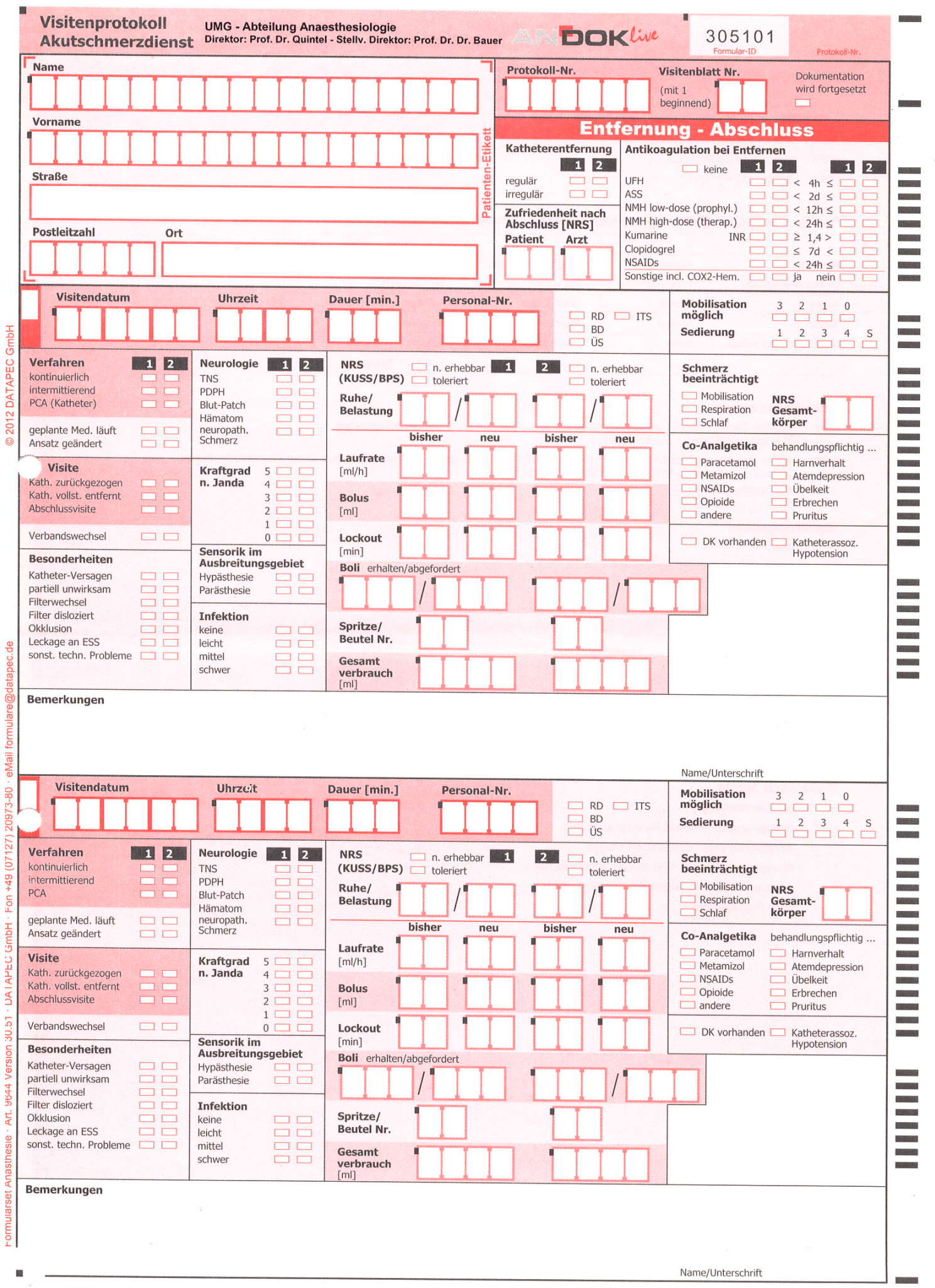




\section{$7 \quad$ Literaturverzeichnis}

Arbeitsgemeinschaft für Osteosynthesefragen 2010, AO-Klassifikation, https://www2.aofoundation.org/wps/portal/surgery; Zugriff am 12.01.2018

Auroy Y, Benhamou D, Bargues L, Ecoffey C, Falissard B, Mercier FJ, Bouaziz H, Samii K, Mercier F (2002): Major complications of regional anesthesia in France: The SOS Regional Anesthesia Hotline Service. Anesthesiology 97, 1274-1280

Avis M, Bond M, Arthur A (1995): Satisfying solutions? A review of some unresolved issues in the measurement of patient satisfaction. Journal of Advanced Nursing 22, 316-322 Barrington MJ, Watts SA, Gledhill SR, Thomas RD, Said SA, Snyder GL, Tay VS, Jamrozik K (2009): Preliminary Results of the Australasian Regional Anaesthesia Collaboration: A Prospective Audit of More Than 7000 Peripheral Nerve and Plexus Blocks for Neurologic and Other Complications. Regional Anesthesia and Pain Medicine 34, 534-541

Beaudoin FL, Nagdev A, Merchant RC, Becker BM (2010): Ultrasound-guided femoral nerve blocks in elderly patients with hip fractures. The American Journal of Emergency Medicine 28, 76-81

Birnbaum K, Prescher A, Hessler S, Heller KD (1997): The sensory innervation of the hip joint-an anatomical study. Surgery Radiology Anatomy 19, 371-375

Brull R, McCartney CJL, Chan VWS, El-Beheiry H (2007): Neurological Complications After Regional Anesthesia: Contemporary Estimates of Risk. Anesthesia \& Analgesia 104, 965-974

Capdevila X, Biboulet P, Bouregba M, Barthelet Y, Rubenovitch J, d'Athis F (1998): Comparison of the Three-in-One and Fascia Iliaca Compartment Blocks in Adults: Clinical and Radiographic Analysis. Anesthesia \& Analgesia 86, 1039-1044

Capdevila X, Biboulet P, Morau D, Bernard N, Deschodt J, Lopez S, d'Athis F (2002): Continuous Three-in-One Block for Postoperative Pain After Lower Limb Orthopedic Surgery: Where Do the Catheters Go?: Anesthesia \& Analgesia 94, 1001-1006

Capdevila X, Pirat P, Bringuier S, Gaertner E, Singelyn F, Bernard N, Choquet O, Bouaziz H, Bonnet F, French Study Group on Continuous Peripheral Nerve Blocks (2005): Continuous peripheral nerve blocks in hospital wards after orthopedic surgery: a multicenter prospective analysis of the quality of postoperative analgesia and complications in 1,416 patients. Anesthesiology 103, 1035-1045

Carr DB, Goudas LC (1999): Acute pain. The Lancet 353, 2051-2058

Chaudet A, Bouhours G, Rineau E, Hamel J-F, Leblanc D, Steiger V, Lasocki S (2016): 
Impact of preoperative continuous femoral blockades on morphine consumption and morphine side effects in hip-fracture patients: A randomized, placebo-controlled study. Anaesthesia Critical Care \& Pain Medicine 35, 37-43

Christos SC, Chiampas G, Offman R, Rifenburg R (2010): Ultrasound-guided three-in-one nerve block for femur fractures. Western Journal Emergergency Mededicine 11, 310-313

Dalens B, Vanneuville G, Tanguy A (1989): Comparison of the fascia iliaca compartment block with the 3-in-1 block in children. Anesthesiology \& Analgesia 69, 705-713

DIVS-Leitlinie 2009 zur Behandlung perioperativer und posttraumatischer Schmerzen. Laubenthal, o. O. 2009

Drake R, Vogl A, Mitchell A, Tibbitts R, Richardson P: Grays Atlas Of Anatomy, 2. Auflage; Churchill Livingstone (Elsevier) Philadelphia/ PA 2015, 292- 368

Duc TA: Postoperative pain control. In: Conroy JM, Dorman BH (Hrsg.): Anesthesia for Orhopedic Surgery. Raven Press, New York 1994, 355_365

Dullenkopf A, Borgeat A (2003): Lokalanaesthetika. Der Anaesthesist 2, 329-340

DVO-Leitlinie 2017 zur Prophylaxe, Diagnostik und Therapie der Osteoporose bei Erwachsenen, http://www.dv-osteologie.org/dvo_leitlinien/dvo-leitlinie-2017; Zugriff am 17.12.2018

Elsharkawy H, Maheshwari A, Farag E, Mariano ER, Rosenquist RW (2016): Development of technologies for placement of perineural catheters. Journal of Anesthesia $\underline{30}, 138-147$

Finlayson BJ, Underhill TJ (1988): Femoral nerve block for analgesia in fractures of the femoral neck. Archives of Emergency Medicine $\underline{5}$, 173-176

Fletcher AK, Rigby AS, Heyes FLP (2003): Three-in-one femoral nerve block as analgesia for fractured neck of femur in the emergency department: A randomized, controlled trial. Annals of Emergency Medicine 41, 227-233

Foss NB (2004): Mortality analysis in hip fracture patients: implications for design of future outcome trials. British Journal of Anaesthesia $\underline{94}$, 24-29

Fournier R, Van Gessel E, Gaggero G, Boccovi S, Forster A, Gamulin Z (1998): Postoperative analgesia with " 3 -in-1" femoral nerve block after prosthetic hip surgery. Canadian Journal of Anaesthesia 45, 34-38

Fredrickson MJ, Danesh-Clough TK (2013): Ultrasound-guided femoral catheter placement: a randomised comparison of the in-plane and out-of-plane techniques. Anaesthesia $\underline{68}, 382-390$

Fredrickson MJ, Ball CM, Dalgleish AJ (2011): Posterior Versus Anterolateral Approach Interscalene Catheter Placement: A Prospective Randomized Trial. Regional Anesthesia and Pain Medicine 36, 125-133 
Ganapathy S, Wasserman RA, Watson JT, Bennett J, Armstrong KP, Stockall CA, Chess DG, MacDonald C (1999): Modified Continuous Femoral Three-in-One Block for Postoperative Pain After Total Knee Arthroplasty. Anesthesia \& Analgesia 89, 1197-1202

Grant S (2001): Continuous peripheral nerve block for ambulatory surgery. Regional Anesthesia and Pain Medicine 26, 209-214

Griffin J, Nicholls B (2010): Ultrasound in regional anaesthesia. Anaesthesia $\underline{65}, 1-12$

Guay J, Parker MJ, Gajendragadkar PR, Kopp S (2016): Anaesthesia for hip fracture surgery in adults. Cochrane Database of Systematic Reviews 2016, https://www.cochranelibrary.com/cdsr/doi/10.1002/14651858.CD000521.pub3/informa tion; Zugriff am 21.01.2018

Hack J, Bliemel C, Ruchholtz S, Bücking B (2015): Anästhesie in der Orthopädie /Unfallchirurgie - Die Versorgung proximaler Femurfrakturen des alten Patienten. AINS Anästhesiologie $\cdot$ Intensivmedizin $\cdot$ Notfallmedizin $\cdot$ Schmerztherapie $\underline{50}$, 250-258

Hartrick CT, Kovan JP, Shapiro S (2003): The Numeric Rating Scale for Clinical Pain Measurement: A Ratio Measure? Pain Practice $\underline{3}$, 310-316

Heavner JE (2007): Local anesthetics: Current Opinion in Anaesthesiology 20, 336-342

Hogan Q (2008): Pathophysiology of Peripheral Nerve Injury During Regional Anesthesia. Regional Anesthesia and Pain Medicine 33, 435-441

Icks A, Haastert B, Wildner M, Becker C, Meyer G (2008): Inzidenz von Hüftfrakturen in Deutschland - Auswertung der Krankenhausdiagnosestatistik 2004. DMW - Deutsche Medizinische Wochenschrift 133, 125-128

Ilfeld BM, Yaksh TL (2009): The End of Postoperative Pain-A Fast-Approaching Possibility? And, if So, Will We Be Ready? Regional Anesthesia and Pain Medicine $\underline{34}$, 85-87

Ilfeld BM, Fredrickson MJ, Mariano ER (2010): Ultrasound-Guided Perineural Catheter Insertion: Three Approaches but Few Illuminating Data. Regional Anesthesia and Pain Medicine 35, 123-126

Ilfeld BM, Sandhu NS, Loland VJ, Madison SJ, Suresh PJ, Mariano ER, Bishop ML, Schwartz AK, Lee DK (2011): Ultrasound-Guided (Needle-in-Plane) Perineural Catheter Insertion: The Effect of Catheter-Insertion Distance on Postoperative Analgesia. Regional Anesthesia and Pain Medicine 36, 261-265

Kanis JA, Odén A, McCloskey EV, Johansson H, Wahl DA, Cooper C (2012): A systematic review of hip fracture incidence and probability of fracture worldwide. Osteoporosis International $\underline{23}, 2239-2256$

Kehlet H, Wilmore DW (2002): Multimodal strategies to improve surgical outcome. The American Journal of Surgery 183, 630-641

Kinjo S, Lim E, Sands LP, Bozic KJ, Leung JM (2012): Does using a femoral nerve block 
for total knee replacement decrease postoperative delirium? BMC Anesthesiology 12, 4

Konnopka A, Jerusel N, König H-H (2009): The health and economic consequences of osteopenia- and osteoporosis-attributable hip fractures in Germany: estimation for 2002 and projection until 2050. Osteoporosis International 20, 1117-1129

Leitlinie Behandlung perioperativer und posttraumatischer Schmerzen s. DIVS-Leitlinie 2009

Leitlinie Osteoporose 2017 s. DVO-Leitlinie 2017

Luger TJ, Kammerlander C, Gosch M, Luger MF, Kammerlander-Knauer U, Roth T, Kreutziger J (2010): Neuroaxial versus general anaesthesia in geriatric patients for hip fracture surgery: does it matter?. Osteoporosis International 21, 555-572

Lynch EP, Lazor MA, Gellis JE, Orav J, Goldman L, Marcantonio ER (1998): The impact of postoperative pain on the development of postoperative delirium. Anesthesiology \& Analgesia $\underline{86}, 781-785$

Marhofer D, Marhofer P, Triffterer L, Leonhardt M, Weber M, Zeitlinger M (2013): Dislocation rates of perineural catheters: a volunteer study. British Journal of Anaesthesia 111, 800-806

Marhofer P, Schrögendorfer K, Koinig H, Kapral S, Weinstabl C, Mayer N (1997): Ultrasonographic guidance improves sensory block and onset time of three-in-one blocks. Anesthiology \& Analgesia $\underline{85}, 854-857$

Morrison RS, Magaziner J, McLaughlin MA, Orosz G, Silberzweig SB, Koval KJ, Siu AL (2003): The impact of post-operative pain on outcomes following hip fracture. Pain 103 , 303-311

Parikh SS, Chung F (1995): Postoperative delirium in the elderly. Anesthsiology \& Analgalgesia $\underline{80}, 1223-1232$

Parker M, Handoll H, Griffiths R, Urwin S (2001): Anaesthesia for hip fracture surgery in adults. Cochrane Database of Systematic Reviews 2001, https://www.cochranelibrary.com/cdsr/doi/10.1002/14651858.CD000521.pub2/referenc es; Zugriff am 21.01.2018

Pham Dang C, Difalco C, Guilley J, Venet G, Hauet P, Lejus C (2009): Various possible positions of conventional catheters around the femoral nerve revealed by neurostimulation. Regional Anesthesia and Pain Medicine 34, 285-289

Richman JM, Liu SS, Courpas G, Wong R, Rowlingson AJ, McGready J, Cohen SR, Wu CL (2006): Does Continuous Peripheral Nerve Block Provide Superior Pain Control to Opioids? A Meta-Analysis. Anesthesia \& Analgesia 102, 248-257 
Ruiz A, Sala-Blanch X, Martinez-Ocón J, Carretero MJ, Sánchez-Etayo G, Hadzic A (2014): Incidence of intraneural needle insertion in ultrasound-guided femoral nerve block: A comparison between the out-of-plane versus the in-plane approaches. Revista Española de Anestesiología y Reanimación 61, 73-77

Salinas F (2004): Prospective comparison of continuous femoral nerve block with nonstimulating catheter placement versus stimulating catheter-guided perineural placement in volunteers. Regional Anesthesia and Pain Medicine 29, 212-220

Schafhalter-Zoppoth I, Zeitz ID, Gray AT (2004): Inadvertent Femoral Nerve Impalement and Intraneural Injection Visualized by Ultrasound. Anesthesia \& Analgesia 627-628

Sharma S, Iorio R, Specht LM, Davies-Lepie S, Healy WL (2010): Complications of Femoral Nerve Block for Total Knee Arthroplasty. Clinical Orthopaedics and Related Research $\underline{468}, 135-140$

Stevens JE, Mizner RL, Snyder-Mackler L (2003): Quadriceps strength and volitional activation before and after total knee arthroplasty for osteoarthritis. Journal of Orthopaedic Research 21, 775-779

Swenson JD, Davis JJ, Stream JO, Crim JR, Burks RT, Greis PE (2015): Local anesthetic injection deep to the fascia iliaca at the level of the inguinal ligament: the pattern of distribution and effects on the obturator nerve. Journal of Clinical Anesthesia 27, 652-657

Tsui B, Dillane D (2006): Needle Puncture Site and a "Walkdown" Approach for ShortAxis Alignment During Ultrasound-Guided Blocks. Regional Anesthesia and Pain Medicine $\underline{31}, 586-587$

Vaurio LE, Sands LP, Wang Y, Mullen EA, Leung JM (2006): Postoperative Delirium: The Importance of Pain and Pain Management. Anesthesia \& Analgesia 102, 1267-1273

Wang A-Z, Gu L, Zhou Q-H, Ni W-Z, Jiang W (2010): Ultrasound-Guided Continuous Femoral Nerve Block for Analgesia After Total Knee Arthroplasty: Catheter Perpendicular to the Nerve Versus Catheter Parallel to the Nerve. Regional Anesthesia and Pain Medicine $\underline{35}, 127-131$

White SM, Griffiths R, Holloway J, Shannon A (2010): Anaesthesia for proximal femoral fracture in the UK: first report from the NHS Hip Fracture Anaesthesia Network. Anaesthesia $\underline{65}, 243-248$

Winnie AP, Ramamurthy S, Durrani Z (1973): The inguinal paravascular technic of lumbar plexus anesthesia: the „3-in-1 block“. Anesthsia \& Analgesia 52, 989-996

Zink W, Graf BM (2003): Toxikologie der Lokalanaesthetika. Der Anaesthesist 르, 11021123 


\section{Danksagung}

Ich möchte mich vielmals bei meinem Doktorvater PD Dr. I. Bergmann und bei Dr. B. Büttner für Thre Hilfe bei der Erstellung dieser Arbeit bedanken. Herrn Prof. Dr. J. Heine aus der Asklepiosklinik Goslar bin ich für die Idee und die Unterstützung an dieser Arbeit sehr verbunden. Mein besonderer Dank gilt auch D. Hansen für die Hilfe bei der Erstellung der Grafiken und der Bildbearbeitung sowie L. Schulte-Güstenberg für ihre Hilfe bei der Durchsicht des Textes. 


\section{Lebenslauf}

Mein Name ist Jan Joschka Jodok Dracklé, und ich wurde am 30.10.1983 in Hamburg als Sohn von Prof. Dr. Dorle Dracklé (Ethnologin) und Dr. Werner Krauß (Ethnologe) geboren.

Von 1990 bis 1994 besuchte ich die Grundschule Kielortalle in Hamburg und von 1991 bis 1992 besuchte ich die Grundschule in Odemira in Portugal. Von 1994 bis 1997 war ich auf der Ida Ehre Gesamtschule in Hamburg und wechselte anschließend an das Gymnasium Eppendorf in Hamburg, das ich am 22.06.2004 mit dem Abitur verließ.

Von 2004 bis 2005 nahm ich an einem einjährigen Engagement in Entwicklungshilfeprojekten in Nicaragua und Guatemala teil, bevor ich zum Wintersemester 2006 das Studium der Humanmedizin in Szeged (Ungarn) in englischer Sprache begann. Nach dem Ersten Abschnitt der Ärztlichen Prüfung studierte ich von 2008 bis 2012 an der Semmelweis Universität AMS Campus Hamburg mit Abschluss der Zweiten Ärztlichen Prüfung am 25.10.2012. Während des Studiums absolvierte ich Auslandspraktika in Nepal und Indien. Nach Erstellung meiner Diplomarbeit mit dem Thema „Latent Autoimmundiabetes in Adults (LADA)“ beendete ich das Studium mit dem Titel dr. med.

Im Oktober 2012 begann ich meine erste Stelle als Assistenzarzt in der Anästhesie an der Asklepios Harzklinik in Goslar. Im Oktober 2015 wechselte ich in die anästhesiologische Abteilung der Universitätsmedizin Göttingen. Im Winter 2017 erlangte ich die Zusatzbezeichnung Notfallmedizin und im Dezember 2018 den Facharzt für Anästhesie. 